\title{
Hydrazinocarben-Metallkomplexe-Synthese aus Allenylidenkomplexen und Hydrazinen, Umlagerung und Cyclisierung
}

\author{
Helmut Fischer ${ }^{*}$, Gerhard Roth \\ Fakultät für Chemie, Universität Konstanz, Postfach 5560, D-78434 Konstanz, Deutschland
}

Eingegangen den 28. Juli 1994

\begin{abstract}
The diarylallenylidene pentacarbonyl complexes $(\mathrm{CO})_{5} \mathrm{M}=\mathrm{C}=\mathrm{C}=\mathrm{C}\left(\mathrm{C}_{6} \mathrm{H}_{4} \mathrm{NMe}_{2}-p\right)_{2}(\mathrm{M}=\mathrm{W}(\mathbf{1})$, $\mathrm{Cr}$ (2)) add 1,2-disubstituted hydrazines RNH-HNR to form alkenyl hydrazino carbene complexes $(\mathrm{CO})_{5} \mathrm{M}=\mathrm{C}\left(\mathrm{C}(\mathrm{H})=\mathrm{C}\left(\mathrm{C}_{6} \mathrm{H}_{4} \mathrm{NMe} \mathrm{e}_{2}-p\right)_{2}\right) \mathrm{NR}-\mathrm{N}(\mathrm{H}) \mathrm{R}(\mathrm{M}=\mathrm{W}$. $\left.\mathrm{R}=\mathrm{Bn}(\mathbf{3 b}),{ }^{\mathrm{i}} \operatorname{Pr}(\mathbf{3 c}),{ }^{\mathrm{c}} \mathrm{Hex}(\mathbf{3 d}) ; \mathrm{M}=\mathrm{Cr}, \mathrm{R}=\mathrm{Me}(\mathbf{4 a}),{ }^{\mathrm{i}} \operatorname{Pr}(\mathbf{4 b})\right)$ in good yield. 3c and $4 \mathrm{~b}$ are formed selectively as $E$-conformers ( $E$ arrangement of $\mathrm{N}_{\beta} \mathrm{HR}$ and $(\mathrm{CO})_{5} \mathrm{M}$ with respect to the $\mathrm{C}$ (carbene) $-\mathrm{N}_{\alpha}$ bond). In contrast, all other derivatives of 3 and 4 are obtained as a mixture of $E / Z$-isomers. On heating, E-3a and $\mathbf{E}-3 \mathbf{b}$ rearrange to give the acrylamidine complexes $(\mathrm{CO})_{5} \mathrm{~W}-$ $\mathrm{NR}=\mathrm{C}(\mathrm{NHR}) \mathrm{C}(\mathrm{H})=\mathrm{C}\left(\mathrm{C}_{6} \mathrm{H}_{4} \mathrm{NMe}_{2}-p\right)_{2}(\mathrm{R}=\mathrm{Me}(\mathbf{5 a}), \mathrm{Bn}(\mathbf{5 b}))$. The structure of complex $5 \mathbf{b}$ was established by $\mathrm{X}$-ray analysis. Acid-catalyzed, the alkenyl hydrazino carbene complexes E-3a, E-3b and $\mathbf{3 c}$ are transformed by intramolecular cyclization into the pyrazolidinylidene complexes $(\mathrm{CO})_{5} \mathrm{~W}=\mathrm{C}-\mathrm{NR}-\mathrm{NR}-\mathrm{C}\left(\mathrm{C}_{6} \mathrm{H}_{4} \mathrm{NMe}_{2}-p\right)_{2}-\mathrm{CH}_{2}\left(\mathrm{R}=\mathrm{Me}(\mathbf{6 a}), \mathrm{Bn}(\mathbf{6 b}),{ }^{\mathrm{i}} \mathrm{Pr}(6 \mathrm{c})\right)$.
\end{abstract}

\section{Zusammenfassung}

Die Diarylallenyliden(pentacarbonyl)komplexe $(\mathrm{CO})_{5} \mathrm{M}=\mathrm{C}=\mathrm{C}=\mathrm{C}\left(\mathrm{C}_{6} \mathrm{H}_{4} \mathrm{NMe}_{2}-\mathrm{p}\right)_{2} \quad(\mathrm{M}=\mathrm{W} \quad(1)$, $\mathrm{Cr}$ (2)) addieren 1,2-disubstituierte Hydrazine RNH-HNR in guten Ausbeuten zu Alkenylhydrazinocarbenkomplexen $(\mathrm{CO})_{5} \mathrm{M}=\mathrm{C}\left(\mathrm{C}(\mathrm{H})=\mathrm{C}\left(\mathrm{C}_{6} \mathrm{H}_{4} \mathrm{NMe}{ }_{2}-\right.\right.$ p) 2 ) NR-N(H)R (M=W, R= Bn (3b), $\left.{ }^{i} \operatorname{Pr}(3 c),{ }^{\mathrm{c}} \mathrm{Hex}(\mathbf{3 d}) ; \mathrm{M}=\mathrm{Cr}, \mathrm{R}=\mathrm{Me}(\mathbf{4 a}),{ }^{\mathrm{i}} \operatorname{Pr}(\mathbf{4 b})\right)$. 3c und 4b entstehen hierbei selektiv in der $E$-Konformation ( $E$-Anordnung von $\mathrm{N}_{\beta} \mathrm{HR}$ und $(\mathrm{CO})_{5} \mathrm{M}$ bezüglich der $\mathrm{C}(\mathrm{Carben})-\mathrm{N}_{\alpha}$-Bindung). Alle anderen Derivate von 3 und 4 werden dagegen als $E / Z$-Isomerengemisch gebildet. E-3a und E-3b lagern sich beim Erwärmen in die Acrylamidinkomplexe $(\mathrm{CO})_{5} \mathrm{~W}-\mathrm{NR}=\mathrm{C}(\mathrm{NHR}) \mathrm{C}(\mathrm{H})=\mathrm{C}\left(\mathrm{C}_{6} \mathrm{H}_{4} \mathrm{NMe}_{2}-p\right)_{2}(\mathrm{R}=\mathrm{Me}(\mathbf{5 a})$, Bn (5b)) um. Die Struktur von $5 \mathbf{b}$ wurde anhand einer Röntgenstrukturanalyse gesichert. Säurekatalysiert cyclisieren die Alkenylhydrazinocarbenkomplexe E-3a, E-3b und 3c zu den

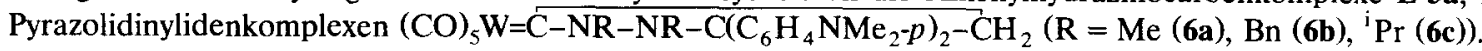

Keywords: Alkenylhydrazinocarbene complexes; X-ray structure analysis; Rearrangement; Chromium; Tungsten

\section{Einleitung}

Allenylidenkomplexe [1] weisen zwei elektrophile Zentren auf, das $\mathrm{C}_{\alpha}$ - und das $\mathrm{C}_{\gamma}$-Atom (GI. (1)).

Demnach sollten sie mit Nukleophilen sowohl über Addition an den metallgebundenen $\left(\mathrm{C}_{\alpha}\right)$ als auch an den terminalen Allenylidenkohlenstoff $\left(\mathrm{C}_{\gamma}\right)$ reagieren können [1c,2,3]. Bislang hat sich gezeigt, daß Stickstoff-Nukleophile ausschließlich am $\mathrm{C}_{\alpha}$-Atom angreifen [2-4]. Bei einfachen $\mathbf{R}^{1} \mathbf{R}^{2} \mathrm{NH}-\mathrm{Nukleophilen}$ (Gl. (2) für $X=N R^{1} R^{2}$ ), resultiert dabei ein Car-

* Corresponding author. benkomplex, dessen Alkenylsubstituent ein weiteres potentielles Reaktionszentrum darstellt.

Durch Umsetzung eines Allenylidenkomplexes mit einem bifunktionellen Nukleophil HY-Z (Gl. (2) für $\mathrm{X}=\mathrm{Y}-\mathrm{Z}$ ) sollten daher Carbenkomplexe zugänglich sein, die sich über eine Folgereaktion zur Darstellung von heterocyclischen Systemen eignen. Diese sind im allgemeinen von großer synthetischer Bedeutung.

Wir berichteten vor kurzem über Reaktionen von Allenylidenkomplexen mit Hydrazinen und über die Isolierung stabiler Hydrazinocarbenkomplexe. Diese erfüllen formal die genannten Voraussetzungen für ein Synthon zur Darstellung von Heterocyclen [3]. Über diese Carbenkomplexklasse ist wenig bekannt [5] und es konnten bislang nur vereinzelte Vertreter dargestellt 


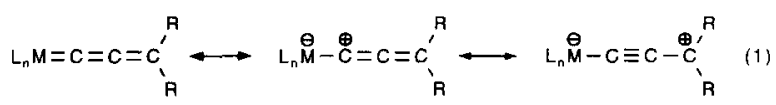

und charakterisiert werden $[3,6]$. Im folgenden sind daher neue Ergebnisse zu diesem Thema zusammengestellt.

\section{Präparative Ergebnisse}

Die bei Raumtemperatur stabilen Allenylidenkomplexe 1 und 2 können als exemplarische Vertreter der Diarylallenylidenchrom- und wolframkomplexe betrachtet werden. Aufgrund der $\pi$-Donorwirkung der p-Aminogruppen sind sie gegenüber nukleophilen Substraten zwar weniger reaktiv als vergleichbare Derivate mit elektronenärmeren aromatischen Substituenten, zeigen aber ein analoges Reaktionsverhalten [3,7].

\subsection{Darstellung von Alkenylhydrazinocarbenkomplexen}

Die Allenylidenkomplexe 1 und $\mathbf{2}$ addieren bei Raumtemperatur 1,2-disubstituierte Hydrazine unter Bildung der Alkenylhydrazinocarbenkomplexe 3 und $\mathbf{4}$. Je nach Nukleophilie der Stickstoffbase und sterischem Anspruch der Substituenten $\mathbf{R}$ ist die Reaktion innerhalb von einigen Minuten bis zu mehreren Stunden abgeschlossen (Gl. (3)). Die Produktkomplexe werden durch den nukleophilen Angriff eines HydrazinStickstoffs am metallgebundenen Allenylidenkohlenstoff $\left(C_{\alpha}\right)$ und nachfolgende irreversible Tautomerisierung zu den Verbindungen 3 und 4 gebildet. Der intermediär auftretende N-Ylidkomplex (vgl. Gl. (2)) wurde spektroskopisch zwar nicht beobachtet, entsprechende Addukte von tertiären Aminen an Carbenkomplexe wurden jedoch schon früher isoliert und charakterisiert [8].

Wie für Aminocarbenkomplexe zu erwarten, liegen auch die Hydrazinocarbenkomplexe 3 und 4 im allgemeinen in zwei isomeren Formen bezüglich der $\mathrm{N}_{\alpha}$ Substituenten vor. Das Verhältnis, in dem die $E$ - und $Z$-Isomeren gebildet werden, hängt bei 3 und 4 , wie auch bei Aminocarbenkomplexen, offensichtlich vom sterischen Anspruch der Hydrazinsubstituenten ab. Die $E$-Konformation wird durch sperrige Gruppen zunehmend begünstigt und entsprechend steigt der

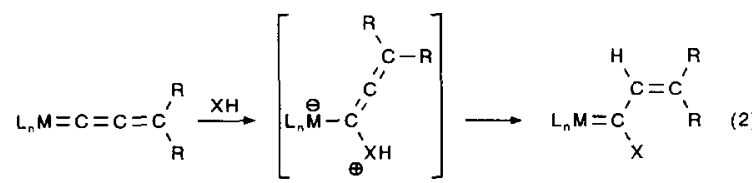

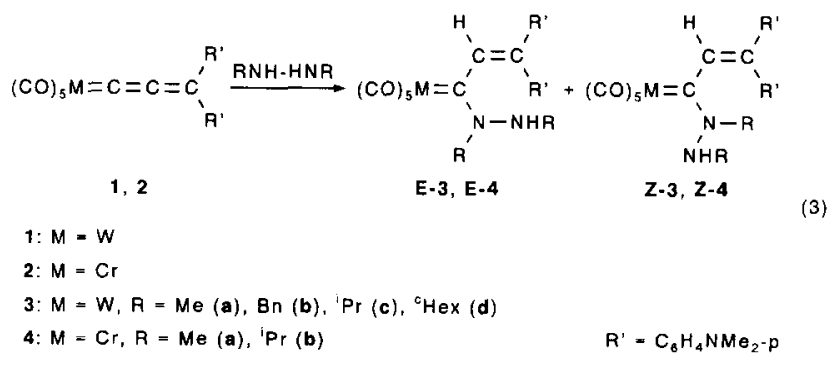

Anteil des $E$-Konformeren im Produktgemisch $(E / Z$ $=1: 0.9$ (3a) [3], $1: 0.6$ (3b), $1: 0$ (3c), $1: 0.8$ (3d), $1: 1$ (4a), $1: 0(\mathbf{4 b}))$. Interessanterweise ist der Energieunterschied der Isomere sowohl für das diisopropylsubstituierte Chrom-, als auch das Wolframderivat so groß, $\mathrm{da} ß$ das postulierte Primäraddukt der Reaktion (vgl. Gl. (2)) jeweils selektiv zum $E$-Konformer 3c bzw. 4 c tautomerisiert.

Die Hydrazinocarbenkomplexe 3 und 4 sind, wie andere Aminocarbenkomplexe, bei Raumtemperatur stabil und zeigen im festen Zustand auch nach Tagen an Luft kaum Zersetzung. Es ist seit langem bekannt, $\mathrm{da} B$ bei Aminocarbenkomplexen die bezüglich der Substituentenstellung am $\mathrm{N}_{\alpha}$-Atom isomeren Verbindungen auch in Lösung meist thermisch nicht ineinander überführbar sind [9]. Diese große Rotationsbarriere wird mit dem hohen Doppelbindungsanteil der $\mathrm{C}($ Carben $)-\mathrm{N}_{\alpha}$-Bindung erklärt. Entsprechendes sollte auch für Hydrazinocarbenkomplexe gelten.

\subsection{Umlagerung von Alkenylhydrazinocarbenkomplexen}

Tatsächlich läßt sich beim Erwärmen der Lösungen von $\mathbf{Z}-3 \mathrm{a}$ und $\mathbf{Z - 3 b}$ in Toluol auf $50^{\circ} \mathrm{C}$ bis $70^{\circ} \mathrm{C}$ über mehrere Stunden keine Isomerisierung zum $E$ Konformeren beobachten. Bei längerem Erhitzen oder auch bei noch höheren Temperaturen tritt lediglich teilweise Zersetzung ein. Die Lösungen der $E$-Isomeren E-3a und E-3b zeigen dagegen ein anderes Verhalten. Unter den gleichen Bedingungen (in Toluol oder THF bei $50^{\circ} \mathrm{C}$ ) sind nach $8 \mathrm{Std}$. bis $16 \mathrm{Std}$. im IR-Spektrum der Reaktionsansätze die Eduktbanden verschwunden. Es erfolgt allerdings keine $E \rightarrow Z$ -

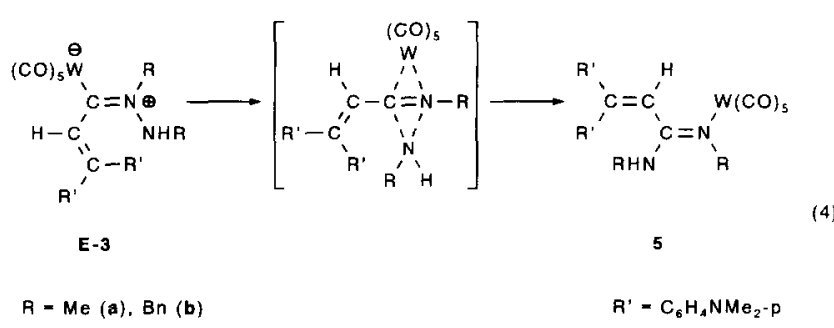


Isomerisierung, sondern eine Umlagerung zu den konstitutionsisomeren Acrylamidinkomplexen 5 (Gl. (4)).

Die Bildung der Verbindungen 5 kann in einfacher Weise verstanden werden, wenn man einen zur Beckmann-Umlagerung [10] analogen Übergangszustand annimmt (Gl. (4)). Dieser wird, ausgehend von der in Gl. (4) gezeigten Grenzform mit einer C(Carben) $-\mathrm{N}_{\alpha}$ Doppelbindung, über die gegenläufige Wanderung der ursprünglich trans-ständigen Gruppen " $\mathrm{W}(\mathrm{CO})_{5}$ " und " $\mathrm{N}_{\beta}$ HR" entlang der $\mathrm{C}\left(\right.$ Carben)- $\mathrm{N}_{\alpha}$-Achse durchlaufen. Aus diesem einfachen Modell ist ersichtlich, daß dieser Austausch bei den Hydrazinocarbenkomplexen mit $Z$-Konformation (W(CO) $)_{5}$ und $\mathrm{N}_{\beta} \mathrm{HR}$ cisständig) nicht möglich ist. Die Reaktionsgeschwindigkeit hängt daneben essentiell von der Art des Substituenten $R$ an der wandernden $N_{\beta}$ HR-Gruppe $a b$. Während für $\mathrm{R}=\mathrm{Me}$ und $\mathrm{Bn}$ die Umlagerung bei $50^{\circ} \mathrm{C}$ nach maximal $16 \mathrm{Std}$. abgeschlossen ist, konnte für $\mathrm{R}={ }^{\mathrm{i}} \mathrm{Pr}$ bei der gleichen Temperatur auch nach $30 \mathrm{Std}$. kein zu 5a,b analoges Produkt isoliert werden.

Als Alternative zu einem Beckmann-Umlagerungsmechanismus ist für die Isomerisierung $\mathbf{E - 3} \rightarrow \mathbf{5}$ eine "dyotrope Umlagerung vom Typ I" [11] in Betracht zu ziehen. Bei dieser orbitalsymmetrie-gesteuerten Reaktion wandern in einem nicht-katalysierten Prozeß zwei $\sigma$-Bindungen gleichzeitig intramolekular. Die beiden wandernden Gruppen vertauschen dabei ihre Stellung miteinander. Hierfür kann ein ähnlicher Übergangszustand wie in Gl. (4) formuliert werden. Nach den geltenden Auswahlregeln wäre auch der sterisch ungünstigere Austausch von cis-ständigen Gruppen möglich. Dieser wird allerdings bei $\mathbf{Z - 3 a}$ und $\mathbf{Z - 3 b}$ nicht beobachtet.

Die Struktur der Acrylamidinkomplexe 5 wurde exemplarisch anhand einer Röntgenstrukturanalyse von 5b belegt (Tabelle 1 und Abb. 1).

Im Festkörper ist der Acrylamidinligand über das freie Elektronenpaar des "Iminostickstoffs" N(1) $\eta^{1}$ koordiniert. Aufgrund der starken Amidinmesomerie besteht ein nur geringer Unterschied in den Bindungslängen des Amidinkohlenstoffs $C(6)$ zum einfach substituierten Stickstoff N(1) (C(6)-N(1) 1.312(8) §) und zum zweifach substituierten Stickstoff N(2) (C(6)-N(2) $1.373(8) \AA$ ). Dementsprechend liegen auch die Methylenkohlenstoffatome C(11) und C(21) der Benzylgruppen sowie $W(1)$ und $C(7)$ näherungsweise in der Amidinebene (Abweichung von der Ebene N(1)-C(6)$\mathrm{N}(2): \mathrm{C}(11)-0.133 \AA, C(21) 0.204 \AA$, W(1) $0.171 \AA$, C(7) $0.100 \AA$ ). Der Alkenylteil des Liganden nimmt wegen der fehlenden Koplanarität nicht an der Mesomerie Teil (Interplanarwinkel zwischen N(1)-C(6)$\mathrm{N}(2)$ und $\mathrm{C}(6)-\mathrm{C}(7)-\mathrm{C}(8)$ : $57.5^{\circ}$, zwischen $\mathrm{C}(6)-\mathrm{C}(7)-$ $\mathrm{C}(8)$ und den $\mathrm{C}_{6} \mathrm{H}_{4} \mathrm{NMe}_{2}$-p-Gruppen: $35.1^{\circ}$ bzw. $48.6^{\circ}$ ). Ähnlich wie bei Carbenkomplexen [8] steht auch bei 5b die Ebene der Substituenten des metallkoordinierten Atoms in einer staggered-Konformation zu den cis-
Tabelle 1

Atomkoordinaten und äquivalente, isotrope thermische Schwingungsparameter $\left(\AA^{2}\right)$ für $\mathbf{5 b}\left(U_{\text {eq }}\right.$ repräsentiert ein Drittel der Spur des orthogonalisierten $U_{i j}$-Tensors)

\begin{tabular}{|c|c|c|c|c|}
\hline Atom & $x$ & $y$ & $z$ & $U_{\mathrm{eq}}$ \\
\hline W(1) & $0.2344(1)$ & $0.1888(1)$ & $0.1172(1)$ & $0.046(1)$ \\
\hline $\mathrm{C}(1)$ & $0.3435(5)$ & $0.0685(8)$ & $0.1044(2)$ & $0.060(3)$ \\
\hline$O(1)$ & $0.4081(4)$ & $0.0092(7)$ & $0.0950(2)$ & $0.096(3)$ \\
\hline $\mathrm{C}(2)$ & $0.3448(9)$ & $0.3109(10)$ & $0.1519(3)$ & $0.110(5)$ \\
\hline$O(2)$ & $0.4013(9)$ & $0.3888(10)$ & $0.1654(3)$ & $0.198(6)$ \\
\hline$C(3)$ & $0.1336(9)$ & $0.3224(8)$ & $0.1281(3)$ & $0.089(4)$ \\
\hline$O(3)$ & $0.0722(7)$ & $0.4008(7)$ & $0.1320(3)$ & $0.136(4)$ \\
\hline$C(4)$ & $0.1179(5)$ & $0.0894(7)$ & $0.0763(3)$ & $0.052(2)$ \\
\hline$O(4)$ & $0.0512(4)$ & $0.0446(6)$ & $0.0514(2)$ & $0.080(2)$ \\
\hline $\mathrm{C}(5)$ & $0.2455(6)$ & $0.2750(7)$ & $0.0556(3)$ & $0.063(3)$ \\
\hline$O(5)$ & $0.2510(6)$ & $0.3187(5)$ & $0.0178(2)$ & $0.091(3)$ \\
\hline$N(1)$ & $0.2247(4)$ & $0.0806(5)$ & $0.1873(2)$ & $0.044(2)$ \\
\hline$C(6)$ & $0.2204(4)$ & $-0.0457(6)$ & $0.1929(2)$ & $0.040(2)$ \\
\hline$N(2)$ & $0.2133(4)$ & $-0.1225(5)$ & $0.1521(2)$ & $0.048(2)$ \\
\hline$C(7)$ & $0.2302(5)$ & $-0.1101(6)$ & $0.2404(2)$ & $0.046(2)$ \\
\hline $\mathrm{C}(8)$ & $0.1673(5)$ & $-0.1943(5)$ & $0.2556(2)$ & $0.040(2)$ \\
\hline$C(11)$ & $0.2222(5)$ & $0.1595(6)$ & $0.2306(2)$ & $0.046(2)$ \\
\hline$C(12)$ & $0.3236(5)$ & $0.2188(6)$ & $0.2533(2)$ & $0.042(2)$ \\
\hline$C(13)$ & $0.3291(6)$ & $0.3400(7)$ & $0.2747(3)$ & $0.060(3)$ \\
\hline$C(14)$ & $0.4200(6)$ & $0.3933(8)$ & $0.2955(3)$ & $0.071(3)$ \\
\hline$C(15)$ & $0.5064(6)$ & $0.3264(7)$ & $0.2964(3)$ & $0.065(3)$ \\
\hline$C(16)$ & $0.5021(6)$ & $0.2044(7)$ & $0.2748(3)$ & $0.069(3)$ \\
\hline$C(17)$ & $0.4113(5)$ & $0.1523(7)$ & $0.2532(3)$ & $0.058(3)$ \\
\hline$C(21)$ & $0.2227(5)$ & $-0.2626(6)$ & $0.1480(2)$ & $0.049(2)$ \\
\hline $\mathrm{C}(22)$ & $0.1781(6)$ & $-0.3018(6)$ & $0.0969(2)$ & $0.052(2)$ \\
\hline$C(23)$ & $0.0780(7)$ & $-0.2925(9)$ & $0.0799(3)$ & $0.080(3)$ \\
\hline$C(24)$ & $0.0402(11)$ & $-0.3255(11)$ & $0.0306(5)$ & $0.121(6)$ \\
\hline$C(25)$ & $0.1042(15)$ & $-0.3692(11)$ & $0.0015(4)$ & $0.135(8)$ \\
\hline$C(26)$ & $0.2003(13)$ & $-0.3761(13)$ & $0.0190(4)$ & $0.135(7)$ \\
\hline $\mathrm{C}(27)$ & $0.2394(9)$ & $-0.3447(9)$ & $0.0665(3)$ & $0.094(4)$ \\
\hline$C(31)$ & $0.1961(5)$ & $-0.2557(6)$ & $0.3043(2)$ & $0.042(2)$ \\
\hline$C(32)$ & $0.2944(5)$ & $-0.2830(7)$ & $0.3253(2)$ & $0.051(2)$ \\
\hline$C(33)$ & $0.3217(5)$ & $-0.3360(7)$ & $0.3715(2)$ & $0.052(2)$ \\
\hline$C(34)$ & $0.2507(5)$ & $-0.3621(7)$ & $0.4001(2)$ & $0.049(2)$ \\
\hline$C(35)$ & $0.1508(5)$ & $-0.3378(6)$ & $0.3788(2)$ & $0.049(2)$ \\
\hline$C(36)$ & $0.1244(5)$ & $-0.2854(6)$ & $0.3329(2)$ & $0.044(2)$ \\
\hline $\mathrm{N}(3)$ & $0.2776(5)$ & $-0.4148(7)$ & $0.4462(2)$ & $0.070(2)$ \\
\hline$C(37)$ & $0.2042(7)$ & $-0.4551(9)$ & $0.4732(3)$ & $0.084(4)$ \\
\hline $\mathrm{C}(38)$ & $0.3804(6)$ & $-0.4477(10)$ & $0.4659(3)$ & $0.096(4)$ \\
\hline$C(41)$ & $0.0682(5)$ & $-0.2273(6)$ & $0.2253(2)$ & $0.039(2)$ \\
\hline$C(42)$ & $0.0048(5)$ & $-0.1339(6)$ & $0.2009(2)$ & $0.041(2)$ \\
\hline$C(43)$ & $-0.0835(5)$ & $-0.1656(6)$ & $0.1703(2)$ & $0.047(2)$ \\
\hline$C(44)$ & $-0.1119(5)$ & $-0.2959(6)$ & $0.1628(2)$ & $0.044(2)$ \\
\hline$C(45)$ & $-0.0502(5)$ & $-0.3906(6)$ & $0.1881(2)$ & $0.045(2)$ \\
\hline$C(46)$ & $0.0382(5)$ & $-0.3571(6)$ & $0.2193(2)$ & $0.045(2)$ \\
\hline N(4) & $-0.2002(5)$ & $-0.3315(6)$ & $0.1315(2)$ & $0.066(2)$ \\
\hline $\mathrm{C}(47)$ & $-0.2145(8)$ & $-0.4663(8)$ & $0.1161(4)$ & $0.105(4)$ \\
\hline$C(48)$ & $-0.2593(7)$ & $-0.2318(9)$ & $0.1033(3)$ & $0.083(4)$ \\
\hline
\end{tabular}

CO-Gruppen des $(\mathrm{CO})_{5} \mathrm{~W}$-Fragments (Interplanarwinkel zwischen $C(1)-C(5)-C(3)$ und $C(6)-N(1)-C(11)$ : $\left.44.9^{\circ}\right)$.

\subsection{Cyclisierung von Alkenylhydrazinocarbenkomplexen}

Diese Ergebnisse lassen darauf schließen, daß eine intramolekulare Cyclisierung von E-Alkenylhydrazinocarbenkomplexen auf thermischem Wege nicht in- 


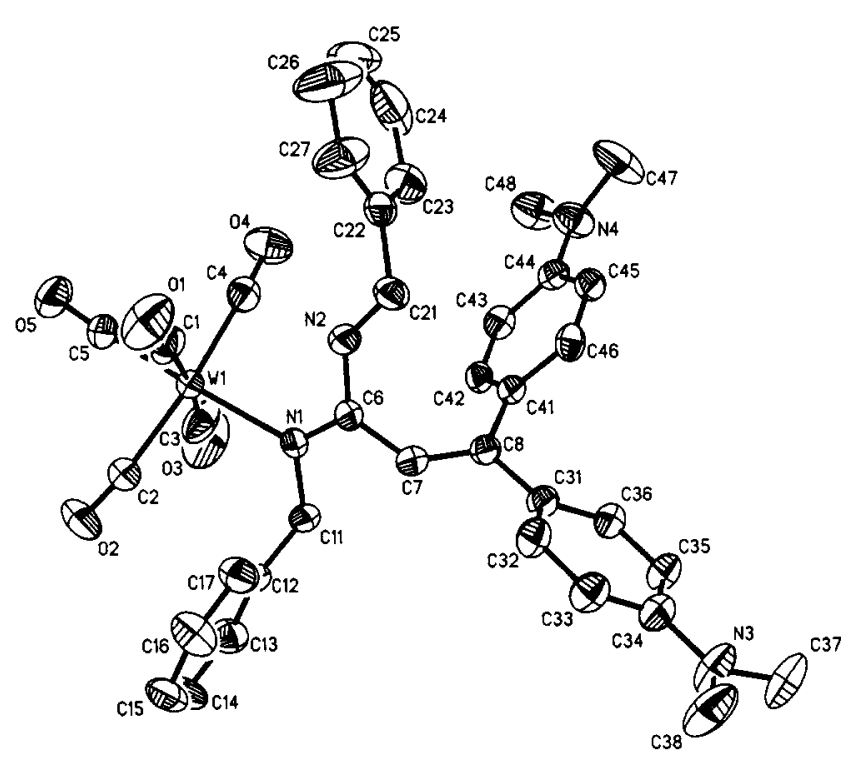

Abb. 1. ORTEP-Darstellung von Komplex 5b im Kristall (ohne HAtome), Ausgewählte Bindungslängen ( $\AA$ ) und -winkel (Grad): $\mathrm{W}(1)-\mathrm{N}(1) 2.276(5), \mathrm{N}(1)-\mathrm{C}(6) 1.312(8), \mathrm{C}(6)-\mathrm{C}(7) 1.465(8), \mathrm{C}(7)$ C(8) 1.345(9), N(1)-C(11) 1.464(8), C(6)-N(2) 1.373(8), N(2)-C(21) $1.454(8), \quad \mathrm{W}(1)-\mathrm{N}(1)-\mathrm{C}(11) \quad 117.0(4), \mathrm{W}(1)-\mathrm{N}(1)-\mathrm{C}(6) \quad 126.8(4)$, C(11)-N(1)-C(6) 116.2(5), N(1)-C(6)-C(7) 123.9(5), N(1)-C(6)-N(2) $117.9(5), \mathrm{C}(6)-\mathrm{C}(7)-\mathrm{C}(8) 128.7(5), \mathrm{C}(6)-\mathrm{N}(2)-\mathrm{C}(21) 129.9(5)$.

duziert werden kann. Es ist bekannt, daß die intermolekulare Addition von schwach nukleophilen (aromatischen) Aminen an vinyloge Carbonsäurederivate ebenfalls nicht thermisch, sondern säurekatalysiert zu guten Ausbeuten an gesättigtem, $\beta$-aminosubstituiertem Reaktionsprodukt führt [12]. In ähnlicher Weise ist die durch Säure katalysierte Addition von Aminen an $\alpha, \beta$-ungesättigte Carbonylverbindungen auch bei der Synthese von Chinolinen nach Skraup verwirklicht [13]. Da diese Systeme aufgrund des Isolobalprinzips mit den hier diskutierten Übergangsmetallkomplexen prinzipiell vergleichbar sind, erscheint diese Reaktionsführung auch im vorliegenden Fall erfolgversprechend.

Werden Lösungen von E-3a bei $40^{\circ} \mathrm{C}$ bzw. von E-3b oder 3c bei Raumtemperatur nach Zugabe von wenig Säure $\left(\mathrm{CF}_{3} \mathrm{COOH}\right.$ oder $\left.\mathrm{HBF}_{4} \cdot \mathrm{Et}_{2} \mathrm{O}\right)$ gerührt, dann lassen sich die Edukte nach einigen Minuten bzw. mehreren Stunden IR-spektroskopisch nicht mehr nachweisen. Aus den Reaktionsansätzen können die fast farblosen Pyrazolidinylidenkomplexe 6 isoliert werden (Gl. (5)).

Die Verbindungen 6 sind sowohl in Lösung, als auch kristallin oxidationsempfindlich und verfärben sich an Luft innerhalb von weniger als einer Stunde rötlich. Entsprechend verhalten sich metallfreie Pyrazolidin-3one. Diese finden wegen ihrer leichten Oxidierbarkeit seit vielen Jahren als photographische Entwickler eine breite Anwendung. Besondere Bedeutung hat hier vor allem 1-Phenylpyrazolidin-3-on ("Phenidon") gewonnen [14].

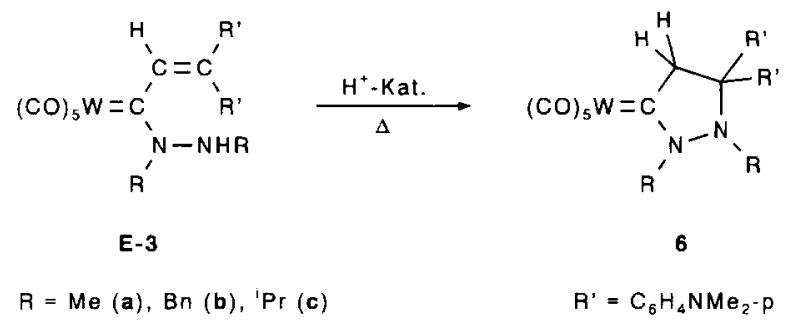

Die NMR-Spektren von 6a-6c zeigen die erwarteten charakteristischen Signale. Die Resonanz der Carbenkohlenstoffe wird beim Übergang von E-3a, b, c $\mathrm{zu}$ den cyclischen Verbindungen 6 nur geringfügig $\mathrm{zu}$ höherem Feld verschoben $(\Delta \delta \approx 12)$ und liegt in dem Bereich, der auch für offenkettige Aminocarbenkomplexe am $(\mathrm{CO})_{5} \mathrm{M}$-Fragment $(\mathrm{M}=\mathrm{Cr}, \mathrm{W})$ gefunden wird [15]. Die Änderung der Alkenylkohlenstoffhybridisierung in $\mathbf{E - 3}$ von $\mathrm{sp}^{2}$ nach $\mathrm{sp}^{3}$ durch die intramolekulare Hydrazinaddition spiegelt sich in der großen Hochfeldwanderung um $\Delta \delta \approx 70$ auf die für 6 beobachteten Werte. Die Resonanz der $\mathrm{CH}_{2}$-Gruppe im Heterocyclus von $\mathbf{6 a - 6 c}$ zeigt sowohl im ${ }^{1} \mathrm{H}-(\delta \approx$ $3.9)$, als auch im ${ }^{13} \mathrm{C}$-NMR $(\delta \approx 74)$ die für einen Carben-Alkylsubstituenten typische Tieffeldlage [15]. Die Unterscheidung des $\mathrm{CH}_{2}$-Signals im ${ }^{13} \mathrm{C}$-NMRSpektrum von demjenigen des zweiten $\mathrm{sp}^{3}$-Ringkohlenstoffs $(\delta \approx 65)$ bei $\mathbf{6 a - 6 c}$ ist durch ein Deuterierungsexperiment (Cyclisierung von E-3a unter $\mathrm{D}^{+}$-Katalyse) möglich.

Sieht man die hier formulierte Cyclisierung der Hydrazinocarbenkomplexe E-3 (Gl. (5)) rückblickend im Zusammenhang mit der bereits zuvor diskutierten thermischen Umlagerung dieser Verbindungen (Gl. (4)), wird anschaulich illustriert, wie stark die Reaktivität und damit das Synthesepotential dieser Komplexklasse durch die äußeren Bedingungen beeinflußt werden kann.

\section{Fazit}

Die Addition von 1,2-disubstituierten Hydrazinen an die Allenylidenkomplexe 1 und 2 führt generell und in guten Ausbeuten zu den bis vor kurzem unbekannten [3] Alkenylhydrazinocarbenkomplexen. Insbesondere die Vertreter dieses Komplextyps mit $E$-Konformation des $\mathrm{N}_{\beta}$ HR-Substituenten zeigen interessante Eigenschaften. Während in einigen Fällen thermisch die Umlagerung zu Acrylamidinkomplexen möglich ist, können sie säurekatalysiert zu Pyrazolidinylidenkomplexen cyclisiert werden. Diese Resultate sollten sich auch auf die Darstellung analoger heterocyclischer Systeme, ausgehend von anderen Dinukleophilen und Allenylidenkomplexen, ausdehnen lassen. 


\section{Experimenteller Teil}

Alle Arbeiten wurden unter Ausschluß von Luft und Feuchtigkeit in einer Inertgasatmosphäre $\left(\mathrm{N}_{2}\right.$ oder Argon) durchgeführt. Die verwendeten Lösungsmittel waren getrocknet $\left(\mathrm{Na}, \mathrm{CaH}_{2}\right)$ und ebenso wie das zur Chromatographie verwendete Kieselgel (Fa. J.T. Baker) mit Stickstoff gesättigt. Die Ausbeuten beziehen sich auf reine Substanzen und sind nicht optimiert. Die NMR-Resonanzen sind auf TMS bezogen und wurden mit Geräten der Fa. Bruker aufgenommen (AC250 und WM250). N,N'-Dibenzyl- [16], N,N'-Diisopropyl- und $\mathrm{N}, \mathrm{N}^{\prime}$-Dicyclohexylhydrazin [17] sowie 1, 2 [7] und E-3a [3] wurden nach bekannten Vorschriften dargestellt. Alle anderen Chemikalien waren handelsüblich ( $\mathrm{Fa}$. Fluka und Fa. Merck).

\subsection{Pentacarbonyl/(E-1-N,N'-dibenzylhydrazino-3,3-bis- p-dimethylaminophenyl)prop-2-enyliden]wolfram (E-3b) und Pentacarbonyl I(Z-1-N,N'-dibenzylhydrazino-3,3-bis- p-dimethylaminophenyl)prop-2-enyliden/wolfram (Z-3b)}

Die blaue Lösung von $4 \mathrm{mmol}(2.40 \mathrm{~g}) 1$ in $60 \mathrm{ml}$ $\mathrm{CH}_{2} \mathrm{Cl}_{2}$ wird mit $5 \mathrm{mmol}(1.06 \mathrm{~g}) \mathrm{N}, \mathrm{N}^{\prime}$-Dibenzylhydrazin versetzt und $1 \mathrm{~h}$ bei Raumtemperatur gerührt, wobei sich die Lösung gelbgrün färbt. Nach Entfernen des Lösungsmittels i. Vak. wird der Rückstand bei $-20^{\circ} \mathrm{C}$ an Kieselgel chromatographiert. Die Elution mit Pentan $/ \mathrm{CH}_{2} \mathrm{Cl}_{2} / \mathrm{Et}_{2} \mathrm{O}(7: 2: 1)$ liefert nach wenig, fast farblosem Vorlauf (W(CO) $)_{6}$ ) eine kurze gelbe Zone, die geringe Mengen $5 b(90 \mathrm{mg}$, siehe Abschnitt 4.7.) enthält. Anschließend läuft eine lange orange Zone aus einem Gemisch von E-3b und Z-3b. Direkt danach eluiert man eine noch längere grünlich gelbe Zone. Diese wird i. Vak. zur Trockne gebracht. Kristallisation aus $70 \mathrm{ml}$ Pentan/ $\mathrm{CH}_{2} \mathrm{Cl}_{2}$ (5:1) liefert E-3b in Form gelboranger Kristalle. Die Mischfraktion wird erneut bei $-20^{\circ} \mathrm{C}$ an Kieselgel chromatographiert. Es wird zunächst mit Pentan $/ \mathrm{CH}_{2} \mathrm{Cl}_{2}(1: 1)$ wenig hellgelber Vorlauf erhalten. Die anschließende Elution mit $\mathrm{CH}_{2} \mathrm{Cl}_{2}$ führt zu einer orangen Zone, die sehr langsam läuft und sich fast über die gesamte Säule erstreckt. Die Zone wird in fünf Fraktionen gesammelt. Die ersten drei Fraktionen liefern nach Entfernen des Solvens und Kristallisation aus $50 \mathrm{ml}$ Pentan/ $\mathrm{CH}_{2} \mathrm{Cl}_{2}(5: 1) \mathbf{Z - 3 b}$ in Form oranger Kristalle. Die restlichen Fraktionen stellen immer noch ein Isomerengemisch dar $(E / Z=1: 2)$, das nicht weiter aufgearbeitet wurde.

\subsection{1. $E-3 b$}

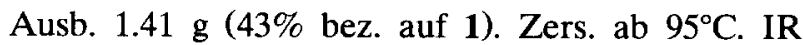
$\left(\mathrm{Et}_{2} \mathrm{O}\right): \nu(\mathrm{CO}) 2060 \mathrm{w}, 1970 \mathrm{vw}, 1928 \mathrm{vs} \mathrm{cm} \mathrm{cm}^{-1}$. ${ }^{1} \mathrm{H}-\mathrm{NMR}$ $\left(\mathrm{CDCl}_{3}\right.$, Raumtemperatur): $\delta=2.98\left(\mathrm{~s}, 6 \mathrm{H}, \mathrm{N}\left(\mathrm{CH}_{3}\right)_{2}\right)$; $2.99\left(\mathrm{~s}, 6 \mathrm{H}, \mathrm{N}\left(\mathrm{CH}_{3}\right)_{2}\right) ; 3.70(\mathrm{dd}, J=12.6$ und $6.9 \mathrm{~Hz}$, $1 \mathrm{H}, \quad \mathrm{N}_{\beta} \mathrm{CH}_{2}$ ); 3.99 (dd, $J=12.6$ und $6.9 \mathrm{~Hz}, 1 \mathrm{H}$,
$\left.\mathrm{N}_{\beta} \mathrm{CH}_{2}\right) ; 5.11\left(\mathrm{~d}, J=14.3 \mathrm{~Hz}, 1 \mathrm{H}, \mathrm{N}_{\alpha} \mathrm{CH}_{2}\right) ; 5.58(\mathrm{~d}$, $\left.J=14.3 \mathrm{~Hz}, 1 \mathrm{H}, \mathrm{N}_{\alpha} \mathrm{CH}_{2}\right) ; 5.87(\mathrm{t}, J=6.9 \mathrm{~Hz}, 1 \mathrm{H}, \mathrm{NH})$; $6.33(\mathrm{~s}, 1 \mathrm{H},=\mathrm{CH}) ; 6.57-6.68(\mathrm{~m}, 4 \mathrm{H}$, aromat. $\mathrm{H})$; 7.01-7.32 (m, $14 \mathrm{H}$, aromat. $\mathrm{H}) .{ }^{13} \mathrm{C}-\mathrm{NMR}\left(\mathrm{CDCl}_{3}\right.$, Raumtemperatur): $\delta=40.2 \quad\left(\mathrm{~N}\left(\mathrm{CH}_{3}\right)_{2}\right) ; 40.3$ $\left(\mathrm{N}\left(\mathrm{CH}_{3}\right)_{2}\right) ; 53.7\left(\mathrm{~N}_{\beta} \mathrm{CH}_{2}\right) ; 65.1\left(\mathrm{~N}_{\alpha} \mathrm{CH}_{2}\right) ; 111.8,112.0$, $126.0,128.1,128.2,128.5,128.8,128.9,129.4,129.6$, $130.7,130.9,133.5,134.2\left(=\mathrm{CH},=C(\text { Aryl })_{2}\right.$, aromat. $\left.\mathrm{C}\right)$; $198.2($ cis-CO, J $(\mathrm{WC})=127 \mathrm{~Hz}) ; 203.2($ trans-CO $)$; 241.2 (Carben-C). Analyse: Gef.: C, 55.93; H, 4.56; N, 6.68. $\mathrm{C}_{38} \mathrm{H}_{36} \mathrm{~N}_{4} \mathrm{O}_{5} \mathrm{~W}$ ber.: $\mathrm{C}, 56.17 ; \mathrm{H}, 4.47 ; \mathrm{N}, 6.90 \%$. Mol.-Gew. 812.5.

\subsection{2. $\mathrm{Z}-3 b$}

Ausb. $0.81 \mathrm{~g}\left(25 \%\right.$ bez. auf 1). Zers. ab $85^{\circ} \mathrm{C}$. IR (Et $\left.{ }_{2} \mathrm{O}\right): \nu(\mathrm{CO}) 2061 \mathrm{w}, 1972 \mathrm{vw}, 1929 \mathrm{vs} \mathrm{cm}{ }^{-1} .{ }^{1} \mathrm{H}-\mathrm{NMR}$ $\left(\mathrm{CDCl}_{3}\right.$, Raumtemperatur): $\delta=2.94\left(\mathrm{~s}, 6 \mathrm{H}, \mathrm{N}\left(\mathrm{CH}_{3}\right)_{2}\right)$; $3.00\left(\mathrm{~s}, 6 \mathrm{H}, \mathrm{N}\left(\mathrm{CH}_{3}\right)_{2}\right) ; 3.94(\mathrm{dd}, J=12.3$ und $7.4 \mathrm{~Hz}$, $1 \mathrm{H}, \mathrm{N}_{\beta} \mathrm{CH}_{2}$ ); 4.08 (dd, $J=12.3$ und $6.4 \mathrm{~Hz}, 1 \mathrm{H}$, $\left.\mathrm{N}_{\beta} \mathrm{CH}_{2}\right) ; 4.17\left(\mathrm{~d}, J=14.9 \mathrm{~Hz}, 1 \mathrm{H}, \mathrm{N}_{\alpha} \mathrm{CH}_{2}\right) ; 5.35(\mathrm{~d}$, $\left.J=14.9 \mathrm{~Hz}, 1 \mathrm{H}, \mathrm{N}_{\alpha} \mathrm{CH}_{2}\right) ; 5.97(\mathrm{t}, J=6.9 \mathrm{~Hz}, 1 \mathrm{H}, \mathrm{NH})$; $6.60-6.76(\mathrm{~m}, 4 \mathrm{H}$, aromat. $\mathrm{H}) ; 6.62(\mathrm{~s}, 1 \mathrm{H},=\mathrm{CH})$; 7.04-7.37 (m, 14H, aromat. H). ${ }^{13} \mathrm{C}-\mathrm{NMR}\left(\mathrm{CDCl}_{3}\right.$, Raumtemperatur): $\delta=40.3 \quad\left(\mathrm{~N}\left(\mathrm{CH}_{3}\right)_{2}\right) ; 40.4$ $\left(\mathrm{N}\left(\mathrm{CH}_{3}\right)_{2}\right) ; 53.0\left(\mathrm{~N}_{\beta} \mathrm{CH}_{2}\right) ; 56.9\left(\mathrm{~N}_{\alpha} \mathrm{CH}_{2}\right) ; 111.7,111.8$, $126.9,127.2,128.0,128.1,128.6,128.7,128.9,129.7$, $131.2,132.6,134.4,134.6,135.7,150.2,150.3(=\mathrm{CH}$, $=C(\text { Aryl })_{2}$, aromat. C); $197.9($ cis $-\mathrm{CO}, J(\mathrm{WC})=126$ $\mathrm{Hz}$ ); 202.9 (trans-CO); 239.0 (Carben-C). Analyse: Gef.: C, 56.21; H, 4.49; N, 6.69. $\mathrm{C}_{38} \mathrm{H}_{36} \mathrm{~N}_{4} \mathrm{O}_{5} \mathrm{~W}$ ber.: $\mathrm{C}$, 56.17; H, 4.47; N, 6.90\%. Mol.-Gew. 812.5.

\subsection{Pentacarbonyl (E-1-N,N'-diisopropylhydrazino-3,3-} bis-p-dimethylaminophenyl)prop-2-enyliden/wolfram (3c)

Analog 4.1. werden $4 \mathrm{mmol}(2.40 \mathrm{~g}) 1$ in $70 \mathrm{ml}$ $\mathrm{CH}_{2} \mathrm{Cl}_{2}$ mit $5 \mathrm{mmol}(0.74 \mathrm{ml}) \mathrm{N}, \mathrm{N}^{\prime}$-Diisopropylhydrazin umgesetzt (Reaktionszeit: 3 d) und chromatographiert. Mit Pentan $/ \mathrm{CH}_{2} \mathrm{Cl}_{2} / \mathrm{Et}_{2} \mathrm{O}$ (ansteigend polar bis $5: 3: 2$ ) wird nach einem hellgelben Vorlauf $\left(\mathrm{W}(\mathrm{CO})_{6}\right)$ die lange gelborange Produktzone eluiert. Diese wird i. Vak. zur Trockne gebracht und liefert aus $80 \mathrm{ml}$ Pentan $/ \mathrm{CH}_{2} \mathrm{Cl}_{2}(3: 1)$ 3c in Form oranger Kristalle. Ausb. $2.55 \mathrm{~g}(89 \%$ bez. auf 1). Zers. ab $115^{\circ} \mathrm{C}$. IR $\left(\mathrm{Et}_{2} \mathrm{O}\right): \nu(\mathrm{CO}) 2059 \mathrm{w}, 1967 \mathrm{vw}, 1926 \mathrm{vs}$, $1916 \mathrm{~m}, \mathrm{sh} \mathrm{cm}{ }^{-1} .{ }^{1} \mathrm{H}-\mathrm{NMR}$ ( $\mathrm{CDCl}_{3}$, Raumtemperatur): $\delta=0.91-0.97\left(\mathrm{~m}, 9 \mathrm{H}, \mathrm{CHCH}_{3}\right) ; 1.30(\mathrm{~d}, J=6.5 \mathrm{~Hz}$, $\left.3 \mathrm{H}, \mathrm{CHCH}_{3}\right) ; 2.96\left(\mathrm{~s}, 6 \mathrm{H}, \mathrm{N}\left(\mathrm{CH}_{3}\right)_{2}\right) ; 2.97(\mathrm{~s}, 6 \mathrm{H}$, $\left.\mathrm{N}\left(\mathrm{CH}_{3}\right)_{2}\right) ; 3.45-3.58\left(\mathrm{~m}, 1 \mathrm{H}, \mathrm{N}_{\beta} \mathrm{CH}\right) ; 4.11(\mathrm{~d}, J=4.7$ $\mathrm{Hz}, 1 \mathrm{H}, \mathrm{NH}$ ); 5.20 (sept, $J=6.5 \mathrm{~Hz}, 1 \mathrm{H}, \mathrm{N}_{\alpha} \mathrm{CH}$ ); $6.64-6.68(\mathrm{~m}, 4 \mathrm{H}$, aromat. $\mathrm{H}) ; 6.78(\mathrm{~s}, 1 \mathrm{H},=\mathrm{CH})$; 7.06-7.18 (m, 4H, aromat. H). ${ }^{13} \mathrm{C}-\mathrm{NMR}\left(\mathrm{CDCl}_{3}\right.$, Raumtemperatur): $\delta=20.0,20.9,21.0,21.3\left(\mathrm{CHCH}_{3}\right)$;

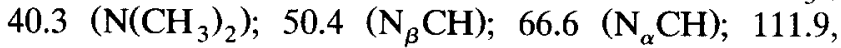
112.1, 126.6, 129.3, 129.5, 130.4, 150.1, 150.3 (aromat. C); $129.9\left(=C(\text { Aryl })_{2}\right) ; 132.7(=\mathrm{CH}) ; 198.4$ (cis-CO, 
$J(\mathrm{WC})=127 \mathrm{~Hz}) ; 204.2($ trans $-\mathrm{CO}) ; 241.5$ (Carben-C). EI-MS: $m / z(\%)=716(13)\left[\mathrm{M}^{+}\right], 576(78)[(\mathrm{M}-$ $5 \mathrm{CO})^{+}$], $334(100)$ [(Carbenligand $\left.-\mathrm{NH}^{\mathrm{i} P r}\right)^{+}$]. Analyse: Gef.: $\mathrm{C}, 50.18 ; \mathrm{H}, 5.25 ; \mathrm{N}, 7.52 . \mathrm{C}_{30} \mathrm{H}_{36} \mathrm{~N}_{4} \mathrm{O}_{5} \mathrm{~W}$ ber.: C, 50.29; H, 5.06; N, 7.82\%. Mol.-Gew. 716.5.

4.3. Pentacarbonyl $\left(\right.$ (E-1-N, $N^{\prime}$-dicyclohexylhydrazino-3,3bis-p-dimethylaminophenyl)prop-2-enyliden Jwolfram (E3d) und Pentacarbonyl/(Z-1-N,N'-dicyclohexylhydrazino-3,3-bis-p-dimethylaminophenyl)prop-2-enyliden]wolfram (Z-3d)

Analog 4.1. werden $4 \mathrm{mmol}(2.40 \mathrm{~g}) 1$ in $50 \mathrm{ml}$ $\mathrm{CH}_{2} \mathrm{Cl}_{2}$ mit $5 \mathrm{mmol}(0.98 \mathrm{~g}) \mathrm{N}, \mathrm{N}^{\prime}$-Dicyclohexylhydrazin umgesetzt (Reaktionszeit: ca. $12 \mathrm{~h}$ ) und chromatographiert. Es wird ansteigend polar mit Pentan/ $\mathrm{CH}_{2} \mathrm{Cl}_{2}$ und schließlich mit $\mathrm{CH}_{2} \mathrm{Cl}_{2}$ eluiert. Die gelborange Produktzone erstreckt sich fast über die gesamte Säule. Nach etwa 2/3 der Fraktion ist eine Zonengrenze schwach zu erkennen. Nach Entfernen des Lösungsmittels der ersten Zone i. Vak. liefert der Rückstand aus $70 \mathrm{ml}$ Pentan $/ \mathrm{CH}_{2} \mathrm{Cl}_{2}(5: 2) \mathbf{E - 3 d}$ in Form oranger Kristalle. Entsprechend erhält man aus der zweiten Zone (Kristallisation aus $50 \mathrm{ml}$ Pentan/ $\left.\mathrm{CH}_{2} \mathrm{Cl}_{2}(5: 2)\right)$ orange Kristalle von $\mathbf{Z - 3 d}$.

\subsection{1. $E-3 d$}

Ausb. $1.40 \mathrm{~g}(44 \%$ bez. auf 1$)$. Zers. ab $120^{\circ} \mathrm{C}$. IR $\left(\mathrm{Et}_{2} \mathrm{O}\right): \nu(\mathrm{CO}) 2059 \mathrm{w}, 1966 \mathrm{vw}, 1925 \mathrm{vs}, 1916 \mathrm{~m}, \mathrm{sh} \mathrm{cm}^{-1}$. ${ }^{1} \mathrm{H}-\mathrm{NMR}\left(\mathrm{CDCl}_{3}\right.$, Raumtemperatur): $\delta=0.83-1.95$ (m br., $20 \mathrm{H}, \mathrm{c}-\mathrm{Hexyl}) ; 2.96\left(\mathrm{~s}, 6 \mathrm{H}, \mathrm{N}\left(\mathrm{CH}_{3}\right)_{2}\right) ; 2.98$ (s, $6 \mathrm{H}$, $\left.\mathrm{N}\left(\mathrm{CH}_{3}\right)_{2}\right) ; 2.97-3.15$ (m br., $1 \mathrm{H}, \mathrm{N}_{\beta} \mathrm{CH}$ ); 4.07 (d, $J=$ $4.0 \mathrm{~Hz}, 1 \mathrm{H}, \mathrm{NH}) ; 4.78-4.95\left(\mathrm{~m}\right.$ br., $\left.1 \mathrm{H}, \mathrm{N}_{\alpha} \mathrm{CH}\right) ; 6.76$ (s, $1 \mathrm{H},=\mathrm{CH}) ; 6.63-7.16(\mathrm{~m}, 8 \mathrm{H}$, aromat. $\mathrm{H}) .{ }^{13} \mathrm{C}-\mathrm{NMR}$ $\left(\mathrm{CDCl}_{3}\right.$, Raumtemperatur): $\delta=24.7,24.8,24.9,25.0$, 25.2, 25.7, 30.2, 30.9, 31.2, 32.2 (c-Hexyl); 40.3 $\left(\mathrm{N}\left(\mathrm{CH}_{3}\right)_{2}\right) ; 58.9\left(\mathrm{~N}_{\beta} \mathrm{CH}\right) ; 74.8\left(\mathrm{~N}_{\alpha} \mathrm{CH}\right) ; 111.9,112.1$, $126.5,129.0,129.5,130.6,150.0,150.3$ (aromat. C); $130.2\left(=C(\text { Aryl })_{2}\right) ; 132.8(=\mathrm{CH}) ; 198.5$ (cis-CO, J(WC) $=127 \mathrm{~Hz}$ ); 204.2 (trans-CO); 241.4 (Carben-C). Analyse: Gef.: C, 54.67; $\mathrm{H}, 5.84 ; \mathrm{N}, 6.65 . \mathrm{C}_{36} \mathrm{H}_{44} \mathrm{~N}_{4} \mathrm{O}_{5} \mathrm{~W}$ ber.: C, 54.28; H, 5.57; N, 7.03\%. Mol.-Gew. 796.6.

\subsubsection{Z-3d}

Ausb. 1.14 g ( $36 \%$ bez. auf 1). Zers. ab $85^{\circ} \mathrm{C}$. IR $\left(\mathrm{Et}_{2} \mathrm{O}\right): \nu(\mathrm{CO}) 2060 \mathrm{w}, 1972 \mathrm{vw}, 1932 \mathrm{vs}, 1926 \mathrm{vs}, \mathrm{sh}, 1904 \mathrm{~m}$ $\mathrm{cm}^{-1} .{ }^{1} \mathrm{H}-\mathrm{NMR}\left(\mathrm{CDCl}_{3}\right.$, Raumtemperatur): $\delta=1.07-$ 1.29 (m br., $8 \mathrm{H}$, c-Hexyl); 1.54-1.85 (m br., $12 \mathrm{H}$, cHexyl); 2.94 (s, 6H, N( $\left.\left.\mathrm{CH}_{3}\right)_{2}\right) ; 2.98\left(\mathrm{~s}, 6 \mathrm{H}, \mathrm{N}\left(\mathrm{CH}_{3}\right)_{2}\right)$; 3.10-3.28 (m br., $1 \mathrm{H}, \mathrm{N}_{\beta} \mathrm{CH}$ ); 4.60-4.69 (m br., $1 \mathrm{H}$, $\left.\mathrm{N}_{\alpha} \mathrm{CH}\right) ; 5.48(\mathrm{~d}, J=9.2 \mathrm{~Hz}, 1 \mathrm{H}, \mathrm{NH}) ; 6.64(\mathrm{~s}, 1 \mathrm{H}$, $=\mathrm{CH}) ; 6.61-7.23(\mathrm{~m}, 8 \mathrm{H}$, aromat. $\mathrm{H}) .{ }^{13} \mathrm{C}-\mathrm{NMR}\left(\mathrm{CDCl}_{3}\right.$, Raumtemperatur): $\delta=25.1,25.5,25.6,25.7,26.0,30.5$, $31.1, \quad 31.3, \quad 32.4$ (c-Hexyl); $40.3\left(\mathrm{~N}\left(\mathrm{CH}_{3}\right)_{2}\right) ; 40.4$ $\left(\mathrm{N}\left(\mathrm{CH}_{3}\right)_{2}\right) ; 58.8\left(\mathrm{~N}_{\beta} \mathrm{CH}\right) ; 67.0\left(\mathrm{~N}_{\alpha} \mathrm{CH}\right) ; 111.7,111.9$, $126.8,129.4,130.3,131.1,150.0,150.1$ (aromat. C);
$131.8\left(=C(\mathrm{Aryl})_{2}\right) ; 133.7(=\mathrm{CH}) ; 198.4$ (cis-CO, $J(\mathrm{WC})$ $=127 \mathrm{~Hz}) ; 202.6$ (trans-CO); 238.8 (Carben-C). Analyse: Gef.: C, 54.13; H, 5.51; N, 7.21. $\mathrm{C}_{36} \mathrm{H}_{44} \mathrm{~N}_{4} \mathrm{O}_{5} \mathrm{~W}$ ber.: C, 54.28; H, 5.57; N, 7.03\%. Mol.-Gew. 796.6.

4.4. Pentacarbonyll(E-1-N, $N^{\prime}$-dimethylhydrazino-3,3-bisp-dimethylaminophenyl)prop-2-enylidenchrom (E-4a) und Pentacarbonyl I(Z-1-N,N'-dimethylhydrazino-3,3bis-p-dimethylaminophenyl)prop-2-enyliden/chrom (Z4a)

Die Umsetzung von $4 \mathrm{mmol}(1.87 \mathrm{~g}) 2$ in $40 \mathrm{ml}$ $\mathrm{CH}_{2} \mathrm{Cl}_{2}$ mit $5 \mathrm{mmol} \mathrm{MeNH}-\mathrm{HNMe}$ (aus $5 \mathrm{mmol}(0.67$ g) N,N'-Dimethylhydrazindihydrochlorid mit $10 \mathrm{mmol}$ $(0.56 \mathrm{~g}) \mathrm{KOH}$ in $3 \mathrm{ml} \mathrm{H}{ }_{2} \mathrm{O}$ freigesetzt) (Reaktionszeit: ca. $12 \mathrm{~h}$ ) und die Chromatographie erfolgen analog 4.1. Die Elution mit Pentan $/ \mathrm{CH}_{2} \mathrm{Cl}_{2} / \mathrm{Et}_{2} \mathrm{O}(7: 2: 1)$ liefert nach einem gelben Vorlauf $\left(\mathrm{Cr}(\mathrm{CO})_{6}\right)$ eine lange orange Produktzone. Diese wird i. Vak. zur Trockne gebracht und der Rückstand aus $45 \mathrm{ml}$ Pentan $/ \mathrm{CH}_{2} \mathrm{Cl}_{2}$ $(4: 1)$ kristallisiert. Es werden zunächst rote Nadeln von Z-4a erhalten. Aus der Mutterlauge kristallisiert nach Entfernen des Lösungsmittels und erneutem Aufnehmen in $25 \mathrm{ml}$ Pentan $/ \mathrm{CH}_{2} \mathrm{Cl}_{2}$ (4:1) E-4a in Form gelber Rhomben (neben wenig Z-4a).

\subsection{1. $E-4 a$}

Ausb. $0.92 \mathrm{~g}\left(42 \%\right.$ bez. auf 2). Zers. ab $85^{\circ} \mathrm{C}$. IR $\left(\mathrm{Et}_{2} \mathrm{O}\right): \nu(\mathrm{CO}) 2052 \mathrm{w}, 1970 \mathrm{vw}, 1928 \mathrm{vs}, 1913 \mathrm{~m}, \mathrm{sh} \mathrm{cm}^{-1}$. ${ }^{1} \mathrm{H}-\mathrm{NMR}\left(\mathrm{CDCl}_{3}\right.$, Raumtemperatur): $\delta=2.50$ (d, $J=$ $\left.6.2 \mathrm{~Hz}, 3 \mathrm{H}, \mathrm{N}_{\beta} \mathrm{CH}_{3}\right) ; 2.97\left(\mathrm{~s}, 6 \mathrm{H}, \mathrm{N}\left(\mathrm{CH}_{3}\right)_{2}\right) ; 2.99(\mathrm{~s}$, $\left.6 \mathrm{H}, \mathrm{N}\left(\mathrm{CH}_{3}\right)_{2}\right) ; 3.82\left(\mathrm{~s}, 3 \mathrm{H}, \mathrm{N}_{\alpha} \mathrm{CH}_{3}\right) ; 6.31(\mathrm{q}, J=6.2$ $\mathrm{Hz}, 1 \mathrm{H}, \mathrm{NH}) ; 6.54(\mathrm{q}, J=1.2 \mathrm{~Hz}, 1 \mathrm{H},=\mathrm{CH}) ; 6.61-7.25$ (m, 8H, aromat. $\mathrm{H}) .{ }^{13} \mathrm{C}-\mathrm{NMR}\left(\mathrm{CDCl}_{3}\right.$, Raumtemperatur): $\delta=36.3 \quad\left(\mathrm{~N}_{\beta} \mathrm{CH}_{3}\right) ; 40.2 \quad\left(\mathrm{~N}\left(\mathrm{CH}_{3}\right)_{2}\right) ; 40.4$ $\left(\mathrm{N}\left(\mathrm{CH}_{3}\right)_{2}\right) ; 46.5\left(\mathrm{~N}_{\alpha} \mathrm{CH}_{3}\right) ; 111.6,111.9,125.8,129.8$, 130.0, 130.7, 150.3 (aromat. C); $128.2(=\mathrm{CH}) ; 133.6$ $\left(=C(\text { Aryl })_{2}\right) ; 217.4$ (cis-CO); 223.3 (trans-CO); 250.1 (Carben-C). EI-MS: $m / z(\%)=528(0.2)\left[\mathrm{M}^{+}\right], 306$ (100) [(Carbenligand $-\mathrm{N}(\mathrm{H}) \mathrm{Me})^{+}$]. Analyse: Gef.: $\mathrm{C}$, 59.73; $\mathrm{H}, 5.82 ; \mathrm{N}, 9.86 . \mathrm{C}_{26} \mathrm{H}_{28} \mathrm{~N}_{4} \mathrm{O}_{5} \mathrm{Cr} \cdot 1 / 3 \mathrm{C}_{5} \mathrm{H}_{12}$ ber.: $\mathrm{C}, 60.16 ; \mathrm{H}, 5.83 ; \mathrm{N}, 10.13 \%$. Mol.-Gew. $528.5+$ 24.5. E-4a konnte bislang nicht vollständig lösungsmittelfrei erhalten werden.

\subsection{2. $Z-4 a$}

Ausb. $0.90 \mathrm{~g}\left(43 \%\right.$ bez. auf 2). Zers. ab $100^{\circ} \mathrm{C}$. IR $\left(\mathrm{Et}_{2} \mathrm{O}\right): \nu(\mathrm{CO}) 2053 \mathrm{w}, 1972 \mathrm{vw}, 1928 \mathrm{vs}, 1916 \mathrm{~m}, \mathrm{sh} \mathrm{cm}^{-1}$. ${ }^{1} \mathrm{H}-\mathrm{NMR}\left(\mathrm{CDCl}_{3}\right.$, Raumtemperatur): $\delta=2.69(\mathrm{~d}, J=$ $\left.6.1 \mathrm{~Hz}, 3 \mathrm{H}, \mathrm{N}_{\beta} \mathrm{CH}_{3}\right) ; 2.95\left(\mathrm{~s}, 6 \mathrm{H}, \mathrm{N}\left(\mathrm{CH}_{3}\right)_{2}\right) ; 2.97(\mathrm{~s}$, $\left.6 \mathrm{H}, \mathrm{N}\left(\mathrm{CH}_{3}\right)_{2}\right) ; 3.10\left(\mathrm{~s}, 3 \mathrm{H}, \mathrm{N}_{\alpha} \mathrm{CH}_{3}\right) ; 6.09(\mathrm{q}, J=6.2$ $\mathrm{Hz}, 1 \mathrm{H}, \mathrm{NH}) ; 6.60(\mathrm{~s}, 1 \mathrm{H},=\mathrm{CH}) ; 6.63-7.23(\mathrm{~m}, 8 \mathrm{H}$, aromat. $\mathrm{H}) .{ }^{13} \mathrm{C}-\mathrm{NMR}\left(\mathrm{CDCl}_{3}\right.$, Raumtemperatur): $\delta=$

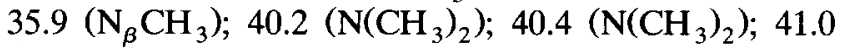
$\left(\mathrm{N}_{\alpha} \mathrm{CH}_{3}\right) ; 111.6,111.8,127.1,129.7,130.3,130.6,150.0$, 150.3 (aromat. C); $131.0(=\mathrm{CH}) ; 134.5\left(=C(\text { Aryl })_{2}\right) ; 217.0$ 
(cis-CO); 223.1 (trans-CO); 245.7 (Carben-C). EI-MS: $m / z(\%)=528$ (n. gef.) $\left[\mathrm{M}^{+}\right], 306(100)$ [(Carbenligand $-\mathrm{N}(\mathrm{H}) \mathrm{Me})^{+}$]. Analyse: Gef.: $\mathrm{C}, 58.50 ; \mathrm{H}, 5.31$; $\mathrm{N}, 10.37 . \mathrm{C}_{26} \mathrm{H}_{28} \mathrm{~N}_{4} \mathrm{O}_{5} \mathrm{Cr}$ ber.: $\mathrm{C}, 59.08 ; \mathrm{H}, 5.34 ; \mathrm{N}$, 10.60\%. Mol.-Gew. 528.5.

4.5. Pentacarbonyl/(E-1-N,N'-diisopropylhydrazino-3,3bis-p-dimethylaminophenyl)prop-2-enyliden/chrom (4b)

Die Umsetzung von $4 \mathrm{mmol}(1.87 \mathrm{~g}) 2$ in $50 \mathrm{ml}$ $\mathrm{CH}_{2} \mathrm{Cl}_{2}$ mit $5 \mathrm{mmol}(0.74 \mathrm{ml}) \mathrm{N}, \mathrm{N}^{\prime}$-Diisopropylhydrazin (Reaktionszeit: $2 \mathrm{~d}$ ) und die Chromatographie erfolgen analog 4.1. Die Elution mit Pentan $/ \mathrm{CH}_{2} \mathrm{Cl}_{2} /$ $\mathrm{Et}_{2} \mathrm{O}$ (ansteigend polar bis 5:3:2) liefert eine lange gelborange Produktzone. Diese wird i. Vak. zur Trockne gebracht und der Rückstand aus $80 \mathrm{ml}$ Pen$\tan / \mathrm{CH}_{2} \mathrm{Cl}_{2} \quad(5: 2)$ kristallisiert. $4 \mathbf{b}$ wird in Form oranger Kristalle erhalten. Ausb. $2.02 \mathrm{~g}(86 \%$ bez. auf 2). Zers. ab $117^{\circ} \mathrm{C}$. IR $\left(\mathrm{Et}_{2} \mathrm{O}\right): \nu(\mathrm{CO}) 2059 \mathrm{w}, 1926 \mathrm{vs}$, $1914 \mathrm{w}, \mathrm{sh} \mathrm{cm}^{-1}$. ${ }^{1} \mathrm{H}-\mathrm{NMR}$ ( $\mathrm{CDCl}_{3}$, Raumtemperatur): $\delta=0.86-0.97\left(\mathrm{~m}, 9 \mathrm{H}, \mathrm{CHCH}_{3}\right) ; 1.31(\mathrm{~d}, J=6.4 \mathrm{~Hz}$, $\left.3 \mathrm{H}, \mathrm{CHCH}_{3}\right) ; 2.96\left(\mathrm{~s}, 6 \mathrm{H}, \mathrm{N}\left(\mathrm{CH}_{3}\right)_{2}\right) ; 2.98(\mathrm{~s}, 6 \mathrm{H}$, $\left.\mathrm{N}\left(\mathrm{CH}_{3}\right)_{2}\right) ; 3.47-3.55\left(\mathrm{~m}, 1 \mathrm{H}, \mathrm{N}_{\beta} \mathrm{CH}\right) ; 4.10(\mathrm{~d}, J=4.3$ $\mathrm{Hz}, 1 \mathrm{H}, \mathrm{NH}$ ); 5.20 (sept, $J=6.4 \mathrm{~Hz}, 1 \mathrm{H}, \mathrm{N}_{\alpha} \mathrm{CH}$ ); $6.64-6.68(\mathrm{~m}, 4 \mathrm{H}$, aromat. $\mathrm{H}) ; 6.89(\mathrm{~s}, 1 \mathrm{H},=\mathrm{CH})$; $7.04-7.16(\mathrm{~m}, 4 \mathrm{H}$, aromat. $\mathrm{H}) .{ }^{13} \mathrm{C}-\mathrm{NMR}\left(\mathrm{CDCl}_{3}\right.$, Raumtemperatur): $\delta=20.1,20.9,21.2\left(\mathrm{CHCH}_{3}\right) ; 40.3$ $\left(\mathrm{N}\left(\mathrm{CH}_{3}\right)_{2}\right) ; 50.3\left(\mathrm{~N}_{\beta} \mathrm{CH}\right) ; 64.4\left(\mathrm{~N}_{\alpha} \mathrm{CH}\right) ; 111.8,112.2$, 126.6, 127.1, 129.4, 130.2, 150.0, 150.2 (aromat. C); $130.0\left(=C(\mathrm{Aryl})_{2}\right) ; 131.8(=\mathrm{CH}) ; 217.4($ cis-CO $) ; 224.2$ (trans-CO); 259.7 (Carben-C). Analyse: Gef.: C, 61.36; $\mathrm{H}, 6.21 ; \mathrm{N}, 9.69 . \mathrm{C}_{30} \mathrm{H}_{36} \mathrm{~N}_{4} \mathrm{O}_{5} \mathrm{Cr}$ ber.: $\mathrm{C}, 61.63 ; \mathrm{H}$, $6.21 ; \mathrm{N}, 9.58 \%$. Mol.-Gew. 584.6.

\subsection{PentacarbonylI $\left(N, N^{\prime}\right.$-dimethyl-3,3-bis-p-dimethyl- aminophenyl)acrylamidin] wolfram (5a)}

Eine Lösung von $0.9 \mathrm{mmol}(0.60 \mathrm{~g}) \mathbf{E - 3 a}$ in $100 \mathrm{ml}$ Toluol wird $8 \mathrm{~h}$ bei $50^{\circ} \mathrm{C}$ gerührt. Die Farbe der orangen Lösung geht mit der Zeit in dunkelrot über. Nach Entfernen des Lösungsmittels i. Vak. wird der Rückstand bei $-20^{\circ} \mathrm{C}$ an Kieselgel chromatographiert. Die mit Pentan $/ \mathrm{CH}_{2} \mathrm{Cl}_{2} / \mathrm{Et}_{2} \mathrm{O}$ (ansteigend polar bis $6: 2: 2$ ) vorauslaufende hellgelbe Zone liefert nach Entfernen des Solvens und Kristallisation aus $20 \mathrm{ml}$ Pentan $/ \mathrm{Et}_{2} \mathrm{O}(8: 2) 5 \mathbf{a}$ in Form gelber Nadeln. Ausb. $0.42 \mathrm{~g}\left(71 \%\right.$ bez. auf E-3a). Schmp. $88-89^{\circ} \mathrm{C}$. IR ( $\left.\mathrm{Et}_{2} \mathrm{O}\right)$ : $\nu(\mathrm{CO}) 2065 \mathrm{w}, 1967 \mathrm{vw}, 1922 \mathrm{vs}, 1897 \mathrm{~m} \mathrm{~cm}^{-1}$. ${ }^{1} \mathrm{H}-\mathrm{NMR}$ $\left(\mathrm{CDCl}_{3}\right.$, Raumtemperatur): $\delta=2.87(\mathrm{~d}, J=5.1 \mathrm{~Hz}$, $\left.3 \mathrm{H}, \mathrm{NHCH}_{3}\right) ; 2.98\left(\mathrm{~s}, 6 \mathrm{H}, \mathrm{N}\left(\mathrm{CH}_{3}\right)_{2}\right) ; 3.01(\mathrm{~s}, 6 \mathrm{H}$, $\left.\mathrm{N}\left(\mathrm{CH}_{3}\right)_{2}\right) ; 3.18\left(\mathrm{~s}, 3 \mathrm{H},=\mathrm{NCH}_{3}\right) ; 5.22(\mathrm{q}, J=5.1 \mathrm{~Hz}$, $1 \mathrm{H}, \mathrm{NH}) ; 5.80(\mathrm{~s}, 1 \mathrm{H},=\mathrm{CH}) ; 6.62-7.30(\mathrm{~m}, 8 \mathrm{H}$, aromat. H). ${ }^{13} \mathrm{C}-\mathrm{NMR}\left(\mathrm{CDCl}_{3}\right.$, Raumtemperatur): $\delta=31.0$ $\left(\mathrm{NHCH}_{3}\right) ; 40.2\left(\mathrm{~N}\left(\mathrm{CH}_{3}\right)_{2}\right) ; 40.3\left(\mathrm{~N}\left(\mathrm{CH}_{3}\right)_{2}\right) ; 50.0$ $\left(=\mathrm{NCH}_{3}\right) ; 109.2(=\mathrm{CH}) ; 111.6,126.0,128.4,129.7,130.0$, 151.0, 151.8 (aromat. $\left.\mathrm{C},=C(\text { Aryl })_{2}\right) ; 167.4(\mathrm{~N}=\mathrm{C}) ; 198.3$
(cis-CO, $J(\mathrm{WC})=130 \mathrm{~Hz}) ; 202.6($ trans-CO). EI-MS $m / z(\%)=660(0.4)\left[\mathrm{M}^{+}\right], 520(13)\left[(\mathrm{M}-5 \mathrm{CO})^{+}\right], 335$ (100) [(Amidinligand $-\mathrm{H})^{+}$]. Analyse: Gef.: $\mathrm{C}$, 48.50; $\mathrm{H}, 4.85 ; \mathrm{N}, 8.32 . \mathrm{C}_{26} \mathrm{H}_{28} \mathrm{~N}_{4} \mathrm{O}_{5} \mathrm{~W} \cdot 1 / 4 \mathrm{C}_{5} \mathrm{H}_{12}$ ber.: $\mathrm{C}$, 48.24; H, 4.61; N, 8.25\%. Mol.-Gew. $660.4+18.0$. 5a konnte bislang nicht vollständig lösungsmittelfrei erhalten werden.

\subsection{Pentacarbonyl/( $N, N^{\prime}$-dibenzyl-3,3-bis-p-dimethyl- aminophenyl)acrylamidin/wolfram (5b)}

Eine Lösung von $1.23 \mathrm{mmol}(1.00 \mathrm{~g}) \mathbf{E}-3 \mathrm{~b}$ in $40 \mathrm{ml}$ THF wird $16 \mathrm{~h}$ bei $50^{\circ} \mathrm{C}$ gerührt. Dabei ist eine Farbvertiefung der orangen Lösung $\mathrm{zu}$ beobachten. Nach Entfernen des Solvens i. Vak. wird der Rückstand bei $-20^{\circ} \mathrm{C}$ an Kieselgel chromatographiert. Die mit Pen$\tan / \mathrm{CH}_{2} \mathrm{Cl}_{2} / \mathrm{Et}_{2} \mathrm{O}(7: 2: 1)$ vorauslaufende gelbe Zone liefert nach Entfernen des Lösungsmittels und Kristallisation aus $30 \mathrm{ml}$ Pentan $/ \mathrm{CH}_{2} \mathrm{Cl}_{2}(15: 1) \mathbf{5 b}$ in Form gelber Nadeln. Ausb. $0.82 \mathrm{~g} \mathrm{(82 \%} \mathrm{bez.} \mathrm{auf} \mathrm{E-3b).}$ Zers. ab $125^{\circ} \mathrm{C}$. IR $\left(\mathrm{Et}_{2} \mathrm{O}\right): \nu(\mathrm{CO}) 2066 \mathrm{w}, 1969 \mathrm{vw}$, $1924 \mathrm{vs}, 1897 \mathrm{~m} \mathrm{~cm}^{-1} .{ }^{1} \mathrm{H}$-NMR $\left(\mathrm{CDCl}_{3}\right.$, Raumtemperatur): $\delta=2.95\left(\mathrm{~s}, 6 \mathrm{H}, \quad \mathrm{N}\left(\mathrm{CH}_{3}\right)_{2}\right) ; 3.02(\mathrm{~s}, 6 \mathrm{H}$, $\left.\mathrm{N}\left(\mathrm{CH}_{3}\right)_{2}\right) ; 4.21-4.51$ (m br., 3H, $\mathrm{NHCH}_{2}$, $=\mathrm{NCH}_{2}$ ); 5.24-5.42 (m br., $\left.1 \mathrm{H},=\mathrm{NCH}_{2}\right) ; 5.58(\mathrm{~s}, 1 \mathrm{H},=\mathrm{CH}) ; 5.99$ (t, $J=5.8 \mathrm{~Hz}, 1 \mathrm{H}, \mathrm{NH}) ; 6.55-6.75(\mathrm{~m}, 4 \mathrm{H}$, aromat. $\mathrm{H})$; 6.97-7.39 (m, $14 \mathrm{H}$, aromat. H). ${ }^{13} \mathrm{C}-\mathrm{NMR}\left(\mathrm{CDCl}_{3}\right.$,

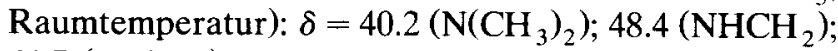
$64.7\left(=\mathrm{NCH}_{2}\right) ; 109.1,111.5,111.8,125.7,126.3,126.7$, $126.9,127.2,127.7,128.1,128.6,128.8,129.8,130.2$, $137.9,140.2,150.6,150.9,151.1\left(=\mathrm{CH},=C(\text { Aryl })_{2}\right.$, aromat. C); $168.0(\mathrm{~N}=\mathrm{C}) ; 197.9($ cis-CO, $J(\mathrm{WC})=117 \mathrm{~Hz})$; 202.1 (trans-CO). Analyse: Gef.: C, 56.13; H, 4.73; N. 6.48. $\mathrm{C}_{38} \mathrm{H}_{36} \mathrm{~N}_{4} \mathrm{O}_{5} \mathrm{~W}$ ber.: $\mathrm{C}, 56.17 ; \mathrm{H}, 4.47$; N, 6.90\%. Mol.-Gew. 812.5.

4.8. Pentacarbonyl/( $N, N^{\prime}$-dimethyl-3,3-bis-p-dimethylaminophenyl)pyrazolidinyliden $/$ wolfram (6a)

Eine Lösung von $0.15 \mathrm{mmol}(0.10 \mathrm{~g}) \mathbf{E - 3 a}$ in $0.5 \mathrm{ml}$ $\mathrm{CDCl}_{3}$ wird mit $5 \mu \mathrm{l}$ Trifluoressigsäure versetzt und 15 min zum Sieden erhitzt. Nach Entfernen des Lösungsmittels i. Vak. wird der Rückstand zweimal mit $30 \mathrm{ml}$ Pentan extrahiert, die erhaltene gelbe Lösung über Kieselgur filtriert und anschließend i. Vak. zur Trockne gebracht. Es verbleibt ein gelbliches Pulver. Ausb. $82 \mathrm{mg}\left(82 \%\right.$ bez. auf E3-a). Schmp. $78-80^{\circ} \mathrm{C}$. IR (Pentan): $\nu$ (CO) 2062w, 1966vw, 1928vs cm ${ }^{-1} .{ }^{1} \mathrm{H}-\mathrm{NMR}$ $\left(\mathrm{CDCl}_{3}\right.$, Raumtemperatur): $\delta=2.39\left(\mathrm{~s}, 3 \mathrm{H}, \mathrm{N}_{\beta} \mathrm{CH}_{3}\right)$; $2.93\left(\mathrm{~s}, 12 \mathrm{H}, \mathrm{N}\left(\mathrm{CH}_{3}\right)_{2}\right) ; 3.73\left(\mathrm{~s}, 3 \mathrm{H}, \mathrm{N}_{\alpha} \mathrm{CH}_{3}\right) ; 3.84$ (d, $\left.J=0.9 \mathrm{~Hz}, 2 \mathrm{H}, \mathrm{CH}_{2}\right) ; 6.62-7.08(\mathrm{~m}, 8 \mathrm{H}$, aromat. $\mathrm{H})$. ${ }^{13} \mathrm{C}-\mathrm{NMR}\left(\mathrm{CDCl}_{3}\right.$, Raumtemperatur): $\delta=37.4$ $\left(\mathrm{N}_{\beta} \mathrm{CH}_{3}\right) ; 40.5 \quad\left(\mathrm{~N}\left(\mathrm{CH}_{3}\right)_{2}\right) ; 41.8 \quad\left(\mathrm{~N}_{\alpha} \mathrm{CH}_{3}\right) ; 66.9$ $\left(C(\mathrm{Aryl})_{2}\right) ; 74.1 \quad\left(\mathrm{CH}_{2}\right) ; 112.0,128.4,130.0,149.7$ (aromat. C); $198.2($ cis-CO, $J(\mathrm{WC})=121 \mathrm{~Hz}) ; 203.0$ (trans-CO); 220.8 (Carben-C). EI-MS: $m / z(\%)=660$ 
(27) $\left[\mathrm{M}^{+}\right], 521(76)\left[(\mathrm{M}-5 \mathrm{CO}+\mathrm{H})^{+}\right], 282(100)$ [((Aryl) $\left.)_{2} \mathrm{C}=\mathrm{N}(\mathrm{H}) \mathrm{Me}\right)^{+}$]. Analyse: Gef.: $\mathrm{C}, 47.81 ; \mathrm{H}, 4.54$; $\mathrm{N}, 8.10 . \mathrm{C}_{26} \mathrm{H}_{28} \mathrm{~N}_{4} \mathrm{O}_{5} \mathrm{~W} \cdot 1 / 8 \mathrm{C}_{5} \mathrm{H}_{12}$ ber.: $\mathrm{C}, 47.78 ; \mathrm{H}$, $4.45 ; \mathrm{N}, 8.36 \%$. Mol.-Gew. $660.4+9.0$. 6a konnte bislang nicht vollständig lösungsmittelfrei erhalten werden.

\subsection{Pentacarbonyl/(N,N'-dibenzyl-3,3-bis-p-dimethyl- aminophenyl)pyrazolidinyliden/wolfram (6b)}

Eine Lösung von $1.5 \mathrm{mmol}(1.21 \mathrm{~g}) \mathbf{E}-3 \mathrm{~b}$ in $120 \mathrm{ml}$ $\mathrm{CH}_{2} \mathrm{Cl}_{2}$ wird mit $0.43 \mathrm{mmol} \mathrm{HBF}_{4}(1 \mathrm{ml}$ einer $0.43 \mathrm{M}$ Lösung von $\mathrm{HBF}_{4} \cdot \mathrm{Et}_{2} \mathrm{O}$ in $\mathrm{Et}_{2} \mathrm{O}$ ) versetzt. Die Farbe der Reaktionslösung ändert sich bei der Säurezugabe von gelborange nach grün. Das Gemisch wird $16 \mathrm{~h}$ bei Raumtemperatur gerührt und ändert dabei die Farbe nach rot. Nach Entfernen des Lösungsmittels i. Vak. wird der Rückstand mit $\mathrm{CH}_{2} \mathrm{Cl}_{2} / \mathrm{Et}_{2} \mathrm{O}(1: 1)$ bei $-20^{\circ} \mathrm{C}$ an Kieselgel chromatographiert. Die vorauslaufende schwach gelb gefärbte Zone wird nach Einengen zur Trockne aus Pentan $/ \mathrm{CH}_{2} \mathrm{Cl}_{2}$ kristallisiert. 6b wird als nahezu farbloses flockiges Pulver erhalten. Ausb. 1.05 g ( $87 \%$ bez. auf E-3b). Zers. ab $150^{\circ} \mathrm{C}$. IR $\left(\mathrm{CH}_{2} \mathrm{Cl}_{2}\right): \nu(\mathrm{CO}) 2062 \mathrm{w}, 1967 \mathrm{vw}, 1923 \mathrm{vs} \mathrm{cm}^{-1} .{ }^{1} \mathrm{H}-$ NMR $\left(\mathrm{CDCl}_{3}\right.$, Raumtemp.): $\delta=2.87 \quad(\mathrm{~s}, 12 \mathrm{H}$, $\left.\mathrm{N}\left(\mathrm{CH}_{3}\right)_{2}\right) ; 3.95$ (s, 2H, Ring- $\left.\mathrm{CH}_{2}\right) ; 3.97(\mathrm{~s}, 2 \mathrm{H}$, $\left.\mathrm{N}_{\beta} \mathrm{CH}_{2}\right) ; 5.18\left(\mathrm{~s}, 2 \mathrm{H}, \mathrm{N}_{\alpha} \mathrm{CH}_{2}\right) ; 6.43-7.34(\mathrm{~m}, 18 \mathrm{H}$, aromat. $\mathrm{H}) .{ }^{13} \mathrm{C}-\mathrm{NMR}\left(\mathrm{CDCl}_{3}\right.$, Raumtemperatur): $\delta=$ $40.4\left(\mathrm{~N}\left(\mathrm{CH}_{3}\right)_{2}\right) ; 54.8\left(\mathrm{~N}_{\beta} \mathrm{CH}_{2}\right) ; 58.8\left(\mathrm{~N}_{\alpha} \mathrm{CH}_{2}\right) ; 65.1$ (C(Aryl) $)_{2}$; 74.0 (Ring- $\mathrm{CH}_{2}$ ); 112.0, 127.4, 128.0, 128.1, 128.2, 128.3, 128.6, 129.7, 133.0, 137.5, 149.5 (aromat. C); $198.0($ cis-CO, $J(\mathrm{WC})=126 \mathrm{~Hz}) ; 202.7($ trans $-\mathrm{CO})$; 228.1 (Carben-C). EI-MS: $m / z(\%)=812(1.5)\left[\mathrm{M}^{+}\right]$, 728 (6) $\left[(\mathrm{M}-3 \mathrm{CO})^{+}\right], 488$ (2.1) [(Pyrazolidinylidenligand $\left.)^{+}\right], 357(23)\left[\left(\mathrm{BnN}=\mathrm{C}\left(\mathrm{C}_{6} \mathrm{H}_{4} \mathrm{NMe}_{2}-p\right)_{2}\right)^{+}\right], 266$ (30) $\left[\left(\mathrm{N}=\mathrm{C}\left(\mathrm{C}_{6} \mathrm{H}_{4} \mathrm{NMe}_{2}-p\right)_{2}\right)^{+}\right], 236(60)\left[\left(\mathrm{N}=\mathrm{C}\left(\mathrm{C}_{6} \mathrm{H}_{4} \mathrm{NMe}_{2^{-}}\right.\right.\right.$ p) $\left.\left.{ }_{2}-2 \mathrm{Me}\right)^{+}\right], 91(100)\left[\mathrm{Bn}^{+}\right]$. Analyse: Gef.: C, 55.38; $\mathrm{H}, 4.52 ; \mathrm{N}, 6.71 . \mathrm{C}_{38} \mathrm{H}_{36} \mathrm{~N}_{4} \mathrm{O}_{5} \mathrm{~W} \cdot 1 / 4 \mathrm{CH}_{2} \mathrm{Cl}_{2}$ ber.: $\mathrm{C}$, 55.10; H, 4.41; N, 6.72\%. Mol.-Gew. 812.5 + 21.3. 6b konnte bislang nicht vollständig lösungsmittelfrei erhalten werden.

\subsection{Pentacarbonyl ( $N, N^{\prime}$-diisopropyl-3,3-bis-p-dimeth- ylaminophenyl)pyrazolidinyliden/wolfram (6c)}

Eine Lösung von $3.5 \mathrm{mmol}(2.50 \mathrm{~g}) 3 \mathrm{c}$ in $70 \mathrm{ml}$ $\mathrm{CH}_{2} \mathrm{Cl}_{2}$ wird mit $0.35 \mathrm{mmol} \mathrm{HBF}_{4}(1 \mathrm{ml}$ einer $0.35 \mathrm{M}$ Lösung von $\mathrm{HBF}_{4} \cdot \mathrm{Et}_{2} \mathrm{O}$ in $\mathrm{Et}_{2} \mathrm{O}$ ) versetzt und $32 \mathrm{~h}$ bei Raumtemperatur gerührt. Die Farbe der Lösung ändert sich währenddessen von orange nach rot. Nach Entfernen des Solvens i. Vak. wird der Rückstand bei $-20^{\circ} \mathrm{C}$ an Kieselgel chromatographiert. Mit Pentan/ $\mathrm{CH}_{2} \mathrm{Cl}_{2} / \mathrm{Et}_{2} \mathrm{O}(7: 2: 1)$ wird, nach wenig gelbem Vorlauf $\left(\mathrm{W}(\mathrm{CO})_{6}\right)$, eine lange gelborange Zone eluiert. Diese liefert nach Entfernen des Lösungsmittels und Kristallisation aus $90 \mathrm{ml}$ Pentan/ $\mathrm{CH}_{2} \mathrm{Cl}_{2}(9: 1)$ 6c in
Form oranger Quader. Ausb. $2.15 \mathrm{~g}$ (86\% bez. auf 3c). Zers. ab $152^{\circ} \mathrm{C}$. IR (Pentan): $\nu$ (CO) 2061w, 1965vw, $1935 \mathrm{~s}, 1926 \mathrm{vs}, 1915 \mathrm{~m} \mathrm{~cm}{ }^{-1} .{ }^{1} \mathrm{H}-\mathrm{NMR}\left(\mathrm{CDCl}_{3}\right.$, Raumtemperatur): $\delta=0.96$ (d, $J=7.0 \mathrm{~Hz}, 6 \mathrm{H}$, $\left.\mathrm{CHCH}_{3}\right) ; 1.52\left(\mathrm{~d}, J=7.0 \mathrm{~Hz}, 6 \mathrm{H}, \mathrm{CHCH}_{3}\right) ; 2.89(\mathrm{~s}$, $\left.12 \mathrm{H}, \mathrm{N}\left(\mathrm{CH}_{3}\right)_{2}\right) ; 3.54$ (sept, $J=7.0 \mathrm{~Hz}, 1 \mathrm{H}, \mathrm{N}_{\beta} \mathrm{CH}$ ); 4.03 (s, $2 \mathrm{H}, \mathrm{CH}_{2}$ ); 4.74 (sept, $J=7.0 \mathrm{~Hz}, 1 \mathrm{H}, \mathrm{N}_{\alpha} \mathrm{CH}$ ); 6.56-6.59 (m, $4 \mathrm{H}$, aromat. $\mathrm{H}) ; 7.23-7.29(\mathrm{~m}, 4 \mathrm{H}$, aromat. H). ${ }^{13} \mathrm{C}-\mathrm{NMR}\left(\mathrm{CDCl}_{3}\right.$, Raumtemperatur): $\delta=$

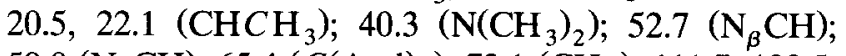
$59.9\left(\mathrm{~N}_{\alpha} \mathrm{CH}\right) ; 65.4\left(C(\text { Aryl })_{2}\right) ; 73.1\left(\mathrm{CH}_{2}\right) ; 111.7,128.5$, 130.1, 149.5 (aromat. C); 198.4 (cis-CO, $J(\mathrm{WC})=127$ $\mathrm{Hz}$ ); 203.4 (trans-CO); 229.6 (Carben-C). EI-MS: $m / z$ $(\%)=716(15)\left[\mathrm{M}^{+}\right], 309(38)\left[\left({ }^{\mathrm{i}} \mathrm{PrN}=\mathrm{C}(\text { Aryl })_{2}\right)^{+}\right], 253$ (100) $\left[\left(\mathrm{HC}(\text { Aryl })_{2}\right)^{+}\right.$]. Analyse: Gef.: C, 50.33; H, 5.03; $\mathrm{N}, 7.82 . \mathrm{C}_{30} \mathrm{H}_{36} \mathrm{~N}_{4} \mathrm{O}_{5} \mathrm{~W}$ ber.: $\mathrm{C}, 50.29 ; \mathrm{H}, 5.06 ; \mathrm{N}$, 7.82\%. Mol.-Gew. 716.5.

\subsection{Röntgenstrukturanalyse von $\mathbf{5 b}$}

$\mathrm{C}_{38} \mathrm{H}_{36} \mathrm{~N}_{4} \mathrm{O}_{5} \mathrm{~W} \cdot 1 / 4 \quad \mathrm{CH}_{2} \mathrm{Cl}_{2} ;$ Molmasse 833.8; Kristallgröße $0.5 \cdot 0.5 \cdot 0.5 \mathrm{~mm}^{3}$ (aus Pentan $/ \mathrm{CH}_{2} \mathrm{Cl}_{2}$ erhalten); Kristallsystem monoklin; Raumgruppe $P 2{ }_{1} / c ; a=13.654(7) \AA, b=10.286(3) \AA, c=27.914(14)$ $\AA, \beta=101.20(4)^{\circ}, V=3846(3) \AA^{3}, Z=4 ; d_{\text {ber }}=1.440 \mathrm{~g}$ $\mathrm{cm}^{-3} ; \mu(\mathrm{Mo}-\mathrm{K} \alpha)=3.145 \mathrm{~mm}^{-1} ; \quad F(000)=1666 ;$ Temp. $-32^{\circ} \mathrm{C}$; Wyckoff-Scan, $2 \theta$-Bereich $4.0^{\circ}$ bis $54.0^{\circ}$, Geschwindigkeit variabel $2.0^{\circ} \mathrm{min}^{-1}$ bis $29.3^{\circ} \mathrm{min}^{-1}$ in $\omega ; 8404$ unabhängige Reflexe, 6284 Reflexe mit $I>$ $4 \sigma(I) ; 445$ verfeinerte Parameter; $R=0.047, R_{\mathrm{w}}=$ 0.056 ; Restelektronendichte $+1.76 /-1.78$ e $\AA^{-3}$. - Ein Einkristall von 5b wurde bei Raumtemperatur in ein Markröhrchen eingeschmolzen. Die Datensammlung und die Ermittlung der Elementarzelle erfolgten mit einem Siemens R3m/V Diffraktometer (Graphitmonochromator, Mo-K $\alpha$-Strahlung, $\lambda=0.71073 \AA$ ). Es wurde eine semi-empirische Absorptionskorrektur (basierend auf 10 Reflexen) durchgeführt. Die Struktur wurde mit Patterson-Methoden (Siemens SHELXTL PLuS (VMS)) gelöst und nach dem Full-Matrix-LeastSquares-Verfahren verfeinert. Dabei wurden die Positionen der Wasserstoffatome in idealer Geometrie berechnet ( $d_{\mathrm{C}-\mathrm{H}}=0.96 \AA$ ) und im "riding model" verfeinert. Alle anderen Atomlagen (außer denen des $\mathrm{zu} 1 / 4$ besetzten Lösungsmittelmoleküls) wurden anisotrop verfeinert. Die Koordinaten der Nicht-Wasserstoffatome sind in Tabelle 1 zusammengestellt. Vollständige Listen der Atomkoordinaten und thermischen Parameter wurden hinterlegt [18].

\section{Dank}

Wir danken dem Fonds der Chemischen Industrie für die großzügige Förderung dieser Untersuchung. 


\section{Literatur und Bemerkungen}

[1] (a) E.O. Fischer, H.J. Kalder, A. Frank, F.H. Köhler und G. Huttner, Angew. Chem., 88 (1976) 683; Angew. Chem., Int. Edn. Engl., 15 (1976) 623; (b) H. Berke, Angew. Chem., 88 (1976) 684; Angew. Chem., Int. Edn. Engl., 15 (1976) 624; (c) Übersicht: M.I. Bruce, Chem. Rev., 91 (1991) 197.

[2] H. Berke, G. Huttner und J. v. Seyerl, Z. Naturforsch., Teil B, 36 (1981) 1277.

[3] H. Fischer, G. Roth, D. Reindl und C. Troll, J. Organomet. Chem., 454 (1993) 133.

[4] F. Stein, M. Duetsch, E. Pohl, R. Herbst-Irmer und A. de Meijere, Organometallics, 12 (1993) 2556.

[5] (a) E.O. Fischer und R. Aumann, Chem. Ber., 101 (1968) 963; (b) A.G.M. Barrett, N.E. Carpenter und M. Sabat, J. Organomet. Chem., 352 (1988) C8.

[6] (a) Y. Ito, T. Hirao und T. Saegusa, J. Organomet. Chem., 131 (1977) 121; (b) H.G. Alt, H.E. Engelhardt und E. Steinlein, $J$. Organomet. Chem., 344 (1988) 321; (c) L.A. Mercando, B.M. Handwerker, H.J. MacMillan, G.L. Geoffroy, A.L. Rheingold und B.E. Owens-Waltermire, Organometallics, 12 (1993) 1559.

[7] H. Fischer, D. Reindl und G. Roth, Z. Naturforsch., Teil B, 49 (1994) 1207.

[8] K.H. Dötz, H. Fischer, P. Hofmann, F.R. Kreissl, U. Schubert und K. Weiss, Transition Metal Carbene Chemistry, Verlag Chemie, Weinheim, 1983.

[9] E. Moser und E.O. Fischer, J. Organomet. Chem., 13 (1968) 387.
[10] Reaktionen [3,5,19], bei denen intermediär gebildete, am $\mathrm{N}_{\alpha}$. Atom unsubstituierte Hydrazinocarbenkomplexe unter Abspaltung eines Amins $\left(\mathrm{N}_{\beta} \mathrm{HR}^{1} \mathrm{R}^{2}\right)$ Nitrilkomplexe bilden, sind daher richtiger als Beckmann-Fragmentierungen [20] zu bezeichnen.

[11] M.T. Reetz, Angew. Chem., 84 (1972) 161; Angew. Chem., Int. Edn. Engl., 11 (1972) 129.

[12] F. Möller, in E. Müller (Hrsg.), Houben-Weyl, Methoden der organischen Chemie, Bd. 11/1, Georg Thieme, Stuttgart, 1957. S. $272 \mathrm{ff}$.

[13] R.H.F. Manske und M. Kulka, Org. Reactions, 7 (1953) 59.

[14] M. Grayson (Ed.), Kirk-Othmer Encyclopedia of Chemical Technology, Vol. 6, 3rd edn., Wiley Interscience, New York, 1979, S. $617 \mathrm{ff}$.

[15] B.E. Mann und B.F. Taylor, ${ }^{13} \mathrm{C}$ NMR Data for Organometallic Compounds, Academic Press, London, 1981.

[16] K.A. Taipale, Chem. Zentralbl., 95 (1924) 902.

[17] R. Renaud und L.C. Leitch, Can. J. Chem., 32 (1954) 545.

[18] Weitere Einzelheiten zur Kristallstrukturanalyse können beim Fachinformationszentrum Karlsruhe, Gesellschaft für wissenschaftlich-technische Information $\mathrm{mbH}$, D-76344 Eggenstein-Leopoldshafen, unter Angabe der Hinterlegungsnummer CSD-58427, der Autoren und des Zeitschriftenzitats angefordert werden.

[19] M.R. Terry, L.A. Mercando, C. Kelley, G.L. Geoffroy, P. Nombel, N. Lugan, R. Mathieu, R.L. Ostrander, B.E. OwensWaltermire und A.L. Rheingold, Organometallics, 13 (1994) 843.

[20] R.E. Gawley, Org. Reactions, 35 (1988) 1. 